\title{
The Development-Based Taxonomy of Stressors and Traumas: An Initial Empirical Validation
}

\author{
Ibrahim Kira1,2 (1) \\ ${ }^{1}$ Center for Cumulative Trauma Studies, Stone Mountain, GA, USA \\ ${ }^{2}$ Center for Stress, Trauma and Resiliency, Georgia State University, Atlanta, GA, USA \\ Email: kiraaref@aol.com
}

How to cite this paper: Kira, I. (2021). The Development-Based Taxonomy of Stressors and Traumas: An Initial Empirical Validation. Psychology, 12, 1575-1614. https://doi.org/10.4236/psych.2021.1210099

Received: September 17, 2021

Accepted: October 23, 2021

Published: October 26, 2021

Copyright $\odot 2021$ by author(s) and Scientific Research Publishing Inc. This work is licensed under the Creative Commons Attribution International License (CC BY 4.0).

http://creativecommons.org/licenses/by/4.0/

\begin{abstract}
The development-based trauma framework (DBTF) proposed a comprehensive taxonomy of stressors and traumas based on the salience of existence and identity development. The goal of this research is to check the validity of some of the DBTF parameters, specifically the trauma types severity (Types I, II, and III), and the six major trauma types (attachment, personal identity, collective identity, role identity, survival and secondary traumas) differential impact and proliferation. We used a sample from 11 Arab countries $(\mathrm{N}=2732)$, age ranged from $18-91$, mean $=26.9$, and $\mathrm{SD}=10.01$, with $69.3 \%$ females. We used measures for cumulative stressors and traumas, PTSD, depression, anxiety, and executive functions. We conducted correlation and path analysis. Correlation explored the differential sizes of association between types I, II, III traumas and mental health and executive function deficits. Path analysis explored the proliferation of and paths between the six major types of trauma and their impact on mental health and cognitive functions. Results indicated that the size of associations of type III trauma and mental health and executive function variable were significantly larger than type II trauma and the same for the difference in the associations between type II and type I, which validate the differential intensity impact of trauma types. Results indicated that attachment traumas have the highest impact on other traumas, followed by early childhood adversities (personal identity trauma) and collective identity traumas, with differential impact on mental health and executive functions. The conceptual, trauma measurement and clinical implications of results were discussed.
\end{abstract}

\section{Keywords}

Type III Traumas, Stressors, Executive Functions, Taxonomy of Trauma, 


\section{Introduction}

The development-based trauma framework (DBTF) that is behind a taxonomy of traumas proposed in the literature (Kira, 2001, 2021; Kira et al., 2008a, 2013a, 2013b, 2014a) redefine traumas as events, sequence of events, or conditions that pose an existential threat to one's physical, personal and social identities. This definition extends the current focus on the physical integrity of the person and the mortality salience model to an identity salience-based definition. It expands the valence of threat to the existential threats to the person's different identities, not only the physical identity death threats. Persons maintain personal autonomy and executive self (Personal identity) and sometimes commit suicide (executing their physical self) if this personal identity is severely violated, which may cause intolerable psychological pain (Joiner, 2005; Shneidman, 1993). They also identify and internalize their group of belonging to the extent that they are ready to die and sacrifice their physical identity for it (e.g., Ashmore et al., 2004; Kira, 2019; Kira et al., 2020b, 2011, 2017; Stryker \& Serpe, 1994). This definition is more in touch with the phenomena of traumatization in real life and real-time. Identity trauma paradigms, personal and collective, are emerging frameworks (e.g., Alexander et al., 2004; Hirschberger, 2018; Kira, 2001, 2006, 2021; Kira et al., 2019a, 2020b; Plotkin-Amrami \& Brunner, 2015). These emerging paradigms are structured and organized within DBTF. Additionally, the DBTF considers traumatic events as part of the global stress dynamics. In real life and real-time, we cannot separate traumatic (acute stressors) from chronic and other non-acute stressors that interact and have a cumulative and global impact on physical, mental, and cognitive functioning.

This definition of traumatic stress is based on the salience of emerging existence (i.e., the salience of the existence of the person's developing identity and her/his executive self) as contrasted to the salience of the physical mortality in the current trauma paradigm. The current trauma paradigm in DSM-IV and DSM-V (APA, 2013) defines trauma, in most part in terms of exposure to actual or threatened death, ignoring the existential threat to identity, personal or social. Mortality and existence are the endpoints of the same continuum. Both mortality and existence are salient human conditions. However, shifting parameters from mortality to existence gave us a more robust conceptual tool to understand the dynamics of traumatization, especially in minorities and children victims of attachment disruptions. Existential threats include physical mortality threats, while physical morality threats do not include all the individual's existential threats. For example, under the current trauma paradigm, the mother's abandonment of the infant may not constitute a trauma because it did not constitute a threat to the physical integrity of the infant. However, the infant's survival as a developing 
person depends, in significant part, on the mother's and caregivers' care. Another entity, for example, an institution, may take care of him/her, and the infant will not physically die from the abandonment. However, his/her emotional and cognitive development may be severely impaired through life span as in foster care experience. Attachment disruptions were found to impact IQ and cognitive functioning (e.g., Kira et al., 2012b). Attachment is a direct existential threat to the infant in the proposed existential paradigm of traumatic stress. The infant's existential survival is entirely dependent on the mother (or the caregiver). According to the DBTF paradigm, attachment is a severe traumatic event that will affect the developing person negatively for the rest of her/his life, as the compelling empirical evidence indicated and will be discussed further in a subsequent section. The same argument applies to discrimination as traumatic stress. Discrimination is not considered trauma in the current trauma paradigm. In the existential (DBTF) paradigm, different discriminations (and intersected discrimination) are existential threats to the person's collective or personal identity. Discrimination and intersected discrimination has severe adverse physical, mental, and cognitive outcomes for the victim, as we will elaborate in a subsequent section. This trauma definition calls for more effective practices for assessing, conceptualizing, and treating traumatized clients.

DBTF-based taxonomy of stressors and trauma include pre-identity (complicated birth, attachment disruptions, early childhood adversities), identity traumas (physical, personal, and social), interdependence (primary vs. secondary, and tertiary), and aging stressors and traumas. Grouping the stressors according to the developmental stage they impact is essential for different reasons. A single trauma may not seriously impact the resilient person; poly-victimization (polytraumatization) or clusters of stressors and traumas that impacts a specific developmental stage and may proliferate across the development is more severe traumatization dynamics than the exposure to a single trauma, that may be an illusion of the last straw that broke the camel's back. It is important to identify stressors that impact each stage and their cumulative dynamics over the life span. In DBTF taxonomy, the severity of stressors type is categorized on a scale from I to III. Type I is a single blow that happened with a limited timescale, such as a car accident. Type II is a sequence of events that happened and stopped, such as sexual abuse. Type III is the continuous traumas/stressors with prolonged time scales that may continue through life. Type III traumas have subtypes or variants that fall under the broader umbrella term of type III trauma, varying in severity and time scale. Discrimination and intersected discrimination represent a type III-a trauma subtype. The type III-b subtype includes exposure to continuous childhood adversities as in the foster care experience. Ongoing intergroup conflicts represent the type III-c trauma sub-type. Chronic community violence represents type III-d, while uncontrolled prolonged medical health conditions such as COVID-19 represent the Type III-e trauma sub-type. Type III traumas can intersect and amplify each other, as in the case of the discrimination (type 
III-a) and COVID-19 (type III-e) intersection (Kira, 2021; Kira et al., 2021c; Solomon \& Heide, 1999; Terr, 1995). The macro-dynamics of intersection, polyvictimization, accumulation, and proliferation, and the interaction among preidentity, identity, and post-identity stressors and traumas determine their total health, mental health, and cognitive impact from a life-course perspective (e.g., Kira, 2001, 2010, 2021; Kira et al., 2012a, 2012c, 2012d, 2013, 2014a, 2014b, 2018a, 2018b, 2019a, 2019b, 2019c, 2019d, 2020d, 2021e; Kira, Barger et al., 2019, 2020).

Different stressors (acute/traumatic and chronic/non-acute) cluster around developmental trajectories and constitute different types. Clustering of the different stressors and traumas around meaningful developmental milestones is essential for conceptual and clinical reasons. Conceptually, a single stressor or trauma can be just the last straw that broke the camel's back or the last rice that tipped the scale. To understand the driving dynamics and increase reproducibility, we may need to adopt an approach that does not test only single associations but compare models representing the outcome-generating mechanisms. Clinically, developing treatment models should be based on the outcome-generating dynamics and not on the last straw or the single rice that tipped the scale.

According to the DBTF framework, there are at least six major stressor clusters/types: Attachment stressors/traumas, personal identity stressors/traumas, collective identity stressors/traumas, physical survival stressors/traumas, role identity stressors/traumas and secondary and tertiary (cross-generation transmission) stressors/traumas. These stressor/trauma types proliferate from the earlier to the subsequent with the earlier (attachment traumas, followed by early childhood adversities and intersected discrimination) proliferate to later adversities with amplification of their impact on health, mental health, and cognitive functioning. We will briefly discuss each stressors cluster/type.

\section{Attachment Stressors/Traumas}

Early abandonment by one parent or both, especially the mother's abandonment in the early three years of life, is an existential threat for the developing infant (Bowlby, 1979). The life of the infants depends entirely on this relationship. Early relational trauma can result in disorganized, ambivalent, avoidant, and unhealthy attachment styles that stay with the person the rest of life, affecting negatively his/her relationship with others, increasing vulnerability to early childhood adversity and other interpersonal traumas and their negative sequelae (e.g., Spinazzola et al., 2018, 2021). Attachment trauma proliferates this way to other trauma types (Kira et al., 2019b). The robust empirical evidence on the adverse effects of unhealthy attachment styles (e.g., for meta-analysis see: Vasileva \& Petermann, 2018), attachment trauma and depression (for meta-analyses see; Spruit et al., 2020; Zheng et al., 2020), and for attachment trauma and PTSD (for meta-analysis see: Woodhouse et al., 2015). There is also robust evidence on the positive effects of secure attachment on resilience, emotion regulation, and wellbeing (e.g., Barnum \& Perrone-McGovern, 2017, for meta-analyses for its link to 
resilience, see: Darling Rasmussen et al., 2019; and for its link to self-regulation see: Pallini et al., 2018). There is evidence that attachment traumas are also associated with deficits in attention and cognitive functioning (for meta-analysis on their association with attention deficits, see: Pallini et al., 2019).

\section{Identity (Personal and Collective) Trauma Model}

Personal identity stressors/traumas: Personal identity traumas are events that challenge the existence of the self as an autonomous, independent agent like physical and sexual abuse or abduction, bullying; emotional abuse; physical neglect, and general maltreatment. Most early childhood adversities belong to this stressors/trauma type. However, this type also includes some adulthood traumas such as rape and domestic violence that question the individual executive ownership of her/his body and self decision-making. Early childhood trauma is associated with adult mental disorders (for meta-analysis, see: McKay et al., 2021). They are associated with depression (for meta-analyses, see Humphreys et al., 2020; Peyrot et al., 2018). Sexual and physical abuse in childhood is associated with depression and anxiety over the life course (For meta-analysis see; Lindert et al., 2014). Early childhood adversities increase the risk of psychosis (for meta-analysis see: Peh et al., 2019; and Varese et al., 2012). Met-analysis found that a significant percentage of children and adolescents develop PTSD after childhood trauma exposure, with those exposed to interpersonal trauma and girls are at particular risk (Trickey et al., 2012). They are also associated with an increased risk of suicide (for meta-analysis, see: Zatti et al., 2017). Childhood adversities are associated with neuropsychological dysfunction and PTSD (for meta-analysis, see: Malarbi et al., 2017).

Intimate partner violence (IPV) is a traumatic experience for the parent victim (and the perpetrator) and their children. A recent meta-analysis found that depression, anxiety, PTSD, antisocial PD, and borderline PD were significantly correlated with IPV victimization and perpetration. Anxiety and PTSD were significantly stronger correlates for victimization than for perpetration, and borderline PD and antisocial PD were significantly stronger correlates for perpetration than for victimization (Spencer et al., 2019).

Collective identity stressors/traumas: Collective identity stressors/traumas are the events or the conditions that threaten the existence or status of one of the person's social identities (Kira, 2020). Different discrimination types that target one or more of the person's social identities are examples of such collective identity stressors and traumas. More than $30 \%$ in the USA report having experienced lifetime discrimination (Kessler et al., 1999). Discrimination is continuous traumatic stress that starts early in adolescence and continues with the person, as long as he/she possesses this identity. The status of self and identity is salient and underlies discriminative acts' dynamics (Kira, 2019; Kira et al., 2019d). Existential anxieties about the person and group statuses that may be the core-specific anxiety in various discrimination types were rarely studied (Kira et al., 2018b, 2019c, 
2020a; Kira, Templin et al., 2012). The extreme lowering of status in the hierarchy can yield vicious dynamics that prevent the person from achieving his/her potentials. Examples of such vicious dynamics are lower self-esteem (de Freitas et al., 2018; Kira et al., 2015; Kucharska, 2018) and self-efficacy (de Freitas et al., 2018), internalized inferiority (for meta-analysis see: Gale et al., 2020), stereotype threat (e.g., Inzlicht et al., 2012), and lower will to live and survive (Kira \& Shuwiekh, 2021; Kira, Özcan et al., 2021d; Kira, Ayna et al., 2021c). Discrimination comprises microaggressions (e.g., implicit and explicit insults to identity status and exclusion) (Sue \& Spanierman, 2020) and macroaggressions such as hate crimes, police brutality, and systemic racism. Macro-aggressions are primary traumas for the directly targeted person and secondary or tertiary (across generations) for those not directly targeted.

Group-based discrimination is associated with poor physical and psychological health (e.g., Pascoe \& Richman, 2009; Williams \& Mohammed, 2009). It was associated with hypertension (Dolezsar et al., 2014), inflammation (e.g., Lewis et al., 2010; Zahodne et al., 2019), cardiovascular events (Everson-Rose et al., 2015), and mortality (Barnes et al., 2008). It was associated with depression, anxiety (Schmitt et al., 2014). The research found that ethnic discrimination is a risk factor for psychotic disorders (Bardol et al., 2020). Discrimination's negative impacts on cognition in different age groups are well documented (e.g., Díaz-Venegas et al., 2016; Zahodne et al., 2021; Kira et al., 2020d).

Intersected discrimination is the cumulative impact of exposure of some or all different types of discrimination in a person or a group. Various discrimination types targeting the same individual add and interact with one another, amplifying their cumulative impact (Abu Ras et al., 2021; McClendon et al., 2021; Seng et al., 2012; for meta-analysis of the mental health impact of intersected discrimination, see Vargas et al., 2020). The non-criterion " $A$ " stressors that include intersected discrimination explained over six-fold of the criterion " $A$ " stressors and fully mediated their effects on PTSD symptoms (Kira et al., 2018a, 2019b).

Physical survival stressors/traumas: Physical survival traumas are events that threaten the physical existence (the life) of the person, such as car, boat, train, airplane accident, a severe accident at work or home, natural disaster, lifethreatening illnesses. Another person has not perpetrated such traumas (Kessler et al., 1995, 2005). However, such non-interpersonal trauma occurs in a social context (Breslau et al., 1999; McLaughlin et al., 2013), and physical survival trauma can happen through interpersonal interaction, like those perpetrate by enemies or during personal and group conflicts and wars, or by criminals.

Role identity stressors/traumas: Role achievement (or role identity) trauma are events that pose existential threats to the person's current and future achievements that structure the person's meaningful role in life (or role identity) that he/she appreciates, such as suddenly get fired from the job, long-term unemployment and chronic school failures, or significant business loss. The harmful 
effects of unemployment on health and mental health are well documented. Unemployment was associated negatively with health status and a significantly higher cardiovascular risk (Noelke et al., 2015), and increased mortality risk (for review and meta-analysis see: Roelfs et al., 2011). It was associated with a greater incidence of suicide (for review and meta-analysis, see: Milner et al., 2014). Longterm unemployed have at least twofold risk of mental illness, particularly depression, anxiety, and other mental disorders (for meta-analysis see: Rönnblad et al., 2019). There is evidence of the association between business performance and suicide (e.g., Abdou et al., 2020). There is empirical evidence of the deleterious effects of the recessions on mental health and suicide rates (e.g., Haw et al., 2015). The harmful effects of school failures on mental and physical health and suicide rates are well documented (e.g., Castellví et al., 2020). School absenteeism was associated with self-harm and suicidal ideation in young people (Epstein et al., 2020).

\section{Interdependence Stressors and Traumas (Secondary and Tertiary Traumas)}

Secondary and tertiary stressors/traumas: Secondary trauma, indirect trauma, vicarious trauma (McCann \& Pearlman, 1990), compassion fatigue (Figley, 1995; Kira, 2001, 2004) are terms used to describe an event that did not happen to you but affected you through different channels and mechanisms. The concept and dynamics of secondary traumas need conceptual clarity. Most research focused on the effects of secondary traumas on professionals working with traumatized clients; however, the concept of secondary trauma and related dynamics apply to professionals as well as all significant others (spouses, parents, children, group of belonging members) exposed to others' traumas directly or through different media outlets. Secondary traumas became even more critical with extended globalization dynamics. There is evidence that females have heightened susceptibility to secondary traumas impact (Baum et al., 2014). One of the mechanisms that transmit such trauma is vicarious empathy (such as in vicarious traumas), as in the therapist's case with traumatized clients. Another mechanism is dependence or systemically connected or enmeshed individuals as in a close relationship; for example, what happened to the mother and witnessed by the child will be secondary trauma for the child, as in the domestic violence case. The phenomena of the "folie adieu" (shared psychosis or induced delusional disorder in a close relationship) are an extreme example of these dynamics (Kira, 2001). Another mechanism of transmission is internalized belonging to a social group. For example, what happened to a person because of his/her group membership, such (e.g., torture or hate crimes or police brutality) terrorizes the rest of the group secondarily; for example, in the cases of hate crimes, police brutality, and critical incidents. Another channel of transmission is the tertiary cross-generational transmission, such as in historical traumas. This channel is described as tertiary trauma, as the transmission can happen through at least three generations. 
Shared traumas are somewhat related to secondary traumas. Shared trauma occurred when two persons or a group of people experienced similar traumatic events (Tosone et al., 2012) or the same traumatic event/s, as is the case of community-wide traumas, such as political terror, natural disaster, or public catastrophe (Ali et al., 2021), and COVID-19 pandemic (Kira et al., 2021a, 2021b, 2021e, 2021f, 2021g). Shared trauma involves unique dynamics, as it involves experiencing the trauma primarily (first hand or directly) and secondarily through its effects on others who shared similar or the same experience.

While the discussed taxonomy is conceptually plausible, it needs empirical validation of its central assumptions. This study empirically tests the assumption of gradient severity between types I, II, and III traumas and some of the dynamics between these six stressors and trauma clusters. Additionally, we used a measure for cumulative stressors and traumas previously developed (Kira et al., 2008a) based on this taxonomy (see Appendix 1). The study conducts an additional test of the measure's validity and its structure. The goal of the current study is to test some of the main parameters of DBTF, precisely the trauma types' paradigm and the trauma primary clusters paradigm. Is type III trauma is the worse considering its impact on mental health and cognitive functioning? Are attachment trauma, personal identity traumas, and collective identity trauma drive the powerful dynamics of trauma proliferation, with attachment trauma the most powerful followed by early childhood and collective identity dynamics?

\section{Hypotheses}

Hypothesis 1: Types II and III traumas have higher significant associations with PTSD, depression, anxiety, and executive function deficits' compared to type I traumas. Type III traumas have a higher significant association with these variables compared to type II traumas.

Hypothesis 2: Attachment traumas, followed by early childhood adversities and intersected discrimination, proliferate to later adversities with amplification of their impact on health, mental health, and cognitive functioning. Attachment trauma proliferates with high size effects on other trauma types, especially on personal and collective identity traumas. It has a high effect size on mental health (depression, anxiety, and PTSD) and executive function deficits, mostly indirectly through its direct impact and proliferation to other traumas types.

Hypothesis 3: Subsequently, personal identity (mostly early childhood adversities) and collective identity trauma (mostly discrimination and intersected discrimination) proliferate to and have moderate size effects on other trauma types (except attachment trauma) and have direct and indirect effects on mental health and executive functions.

Hypothesis 4: Role identity trauma proliferates to collective identity and has significant but a small effect size on mental health and executive functions variables.

Hypothesis 5: The other trauma types (survival and secondary traumas) have small but significant effect sizes on mental health and executive functions. 


\section{Methods}

1) Participants: The sample included $N=2732$ adults from 11 Arab countries (Algeria, Iraq, Jordan, Kuwait, Lebanon, Libya, Palestinian, Saudi Arabia, Sudan, UAE, and Egypt). Age ranged from $18-91$, mean $=26.9$, and $\mathrm{SD}=10.01$, with 69.3\% Females, $97.3 \%$ were Muslims and 1.4\% Christians, .3\% Jewish, $1 \%$ other. For education, $1.4 \%$ reading and writing level, $8.7 \%$ high school level, $81.6 \%$ college level, and $8.3 \%$ graduate studies level. For marital status, $63.2 \%$ single $31 . \%$ married, $3.2 \%$ widowed, $.9 \%$ divorced. For employment, $27.2 \%$ work with the government, $58.1 \%$ Students, $6.2 \%$ private business, $2.2 \%$ retired, and $5.8 \%$ unemployed. For socioeconomic status (SES), .7\% reported very low SES, $1.2 \%$ low SES, $81.3 \%$ in the middle, $14.5 \%$ high, and $2.3 \%$ reported very high SES. For country representation, $30.4 \%$ from Egypt $(\mathrm{N}=831), 10.1 \%$ from Kuwait $(\mathrm{N}=$ 275), $10.3 \%$ from Jordan $(\mathrm{N}=281), 7.6 \%$ from Algeria $(\mathrm{N}=207), 9.7 \%$ from Saudi Arabia $(\mathrm{N}=265)$, and $8.3 \%$, from Iraq $(\mathrm{N}=226)$, and $6.6 \%$ from Palestine $(\mathrm{N}=180), 6.1 \%$ from Libya $(\mathrm{N}=167), 5.1 \%$ from Lebanon $(\mathrm{N}=142), 3.6 \%$ from Sudan $(\mathrm{N}=98)$, and $2.2 \%$ from UAE $(\mathrm{N}=60)$.

2) Procedures: A team of three core researchers designed the study. The field study team of graduate students from the participating Arab countries administered the questionnaires to participants from January to March of 2021. We chose the eleven Arabic countries: Algeria, Iraq, Jordan, Kuwait, Lebanon, Libya, Palestine, Saudi Arabia, Sudan, UAE, and Egypt, to represent the 22 Arab countries (50\%). We used Google Drive and developed a survey link. Once the participant completed the survey, it was sent anonymously to Gmail then downloaded to the Excel file. All questionnaires were administered individually to participants. The questionnaire was administered face to face $=17.5 \%$ and online $=82.5 \%$. Participation was voluntary with built-in informed consent; each person took approximately 20 minutes to complete the full questionnaire. The sponsoring university IRB approved the research protocol as part of a crosscultural study of the impact of COVID-19.

\section{Measures}

1) Cumulative stressors and traumas scale (CTS-S-36 items; Kira et al., 2008a): CST-S was developed to measure stressors and traumas identified in the development-based stressors and traumas framework (DBTF) (e.g., Kira, 2001, 2019, 2021; Kira, Shuwiekh et al., 2018, 2019a). The scale is intended to be a comprehensive measure of cumulative stressors and trauma exposure and measures different stressors and trauma categories and their severity types (type I, II, and III). The current alpha of cumulative stressors and traumatic occurrence is .97 . The alphas for trauma types 1 , II, and III are 84,86 , and .88 , respectively. A detailed description of the scale and instruction of its use and scoring is found in Appendix 1.

2) Posttraumatic Stress Disorder Checklist for DSM-5 (PCL-V; Blevins et al., 2015): PCL-5 is a scale that measures PTSD according to DSM-5 criteria. It 
includes 20-questions. Each question is scored on a five-point scale with "0," signifying "not at all," and 4 signifying "extremely." PCL-5 cut-off scores range for probable PTSD is $31-33$. The measure was previously translated to Arabic and validated (Ibrahim et al., 2018). Cronbach's alpha reliability of the scale in the current study was 95 .

3) Generalized Anxiety Disorder-7 (GAD-7; Spitzer et al., 2006): GAD-7 is a 7-item self-report scale that assesses generalized anxiety. Questions are scored on a 4-point scale with (0) indicating "does not exist" and (3) indicating that the symptom exists "nearly every day." A cut-off point of 15 signifies severe GAD. The measure has a specificity of $82 \%$ and a sensitivity of $89 \%$. The measure's High scores had been strongly associated with functional impairment in multiple domains (Spitzer et al., 2006). The measure was previously translated to Arabic and validated (Sawaya et al., 2016). Cronbach's alpha for the measure in the current study was .92.

4) Patient Health Questionnaire-9 (PHQ-9; Kroenke et al., 2001) is a 9-item measure that assesses depression severity. Items are scored on a 4-point scale with (0) signifying "does not exist" and (3) indicating that the behavior is happening "nearly every day." A cut-off score ranges from 15 - 19, signifying moderate to severe depression, while a score of 20 and above indicates severe depression. The Arabic version of PhQ-9 was previously translated and validated (Sawaya et al., 2016). Alpha reliability for the measure in the current study was .88 .

5) Post-cumulative trauma disorders (P-CTD) Scale (Kira et al, 2012c). The 16-item measure was developed in five community and clinic samples of African American and Arab adolescents and adults. It is based on the idea that cumulative stressors and traumas may yield a complex picture beyond the description of the traditional diagnostic categories, especially in minorities and refugees. The CTD is an index measure for comorbid clustered syndromes that assesses 14 different symptoms: depression, anxiety, somatization, dissociation, auditory and visual hallucinations, avoidance of being with people, paranoid ideations, concentration and memory deficits, loss of self-control, feeling suicidal, and feeling like hurting self and have a plan to kill self. The exploratory and confirmatory factor analysis results found support for four factors: Executive control deficits, Suicidality, Dissociation/Psychosis, and Depression/Anxiety/Somatization Comorbidity. The measure has good reliability (ranging from .85 and .98). Testretest reliability in a 6-week interval was .76. Several studies offered support for its predictive and criterion validity. Different kinds of traumas and cumulative trauma accounted for significant variance as predictors of cumulative trauma disorders (Kira, Templin et al., 2012). For the severity of symptoms, the authors recommended a cut-off point score of $\geq 22$ for the total score, $\geq 9$ for comorbid depression/anxiety/somatization Subscale, $\geq 9$ for comorbid psychotic, and dissociation Subscale. For suicidality symptoms, cluster $\geq 5$ is the cut-off point score. Alpha for the full scale in the current study was .93, and for its four subscales was .83 for comorbidity, 85 for suicidality, .78 for psychosis/dissociation, 
and .75 for executive control deficits.

6) The Adult Executive Functioning Inventory (ADEXI; Holst \& Thorell, 2018) was used to investigate executive functioning deficits. The ADEXI is a 14 -item scale that measures working memory deficits (9 items) (e.g., "I have difficulty remembering lengthy instructions" and inhibition deficits (5 items) (e.g., "I tend to do things without first thinking about what could happen"). The participant is asked to rate the statement on a scale from 1 to 5 , with " 1 " indicates that it is definitely not true, and " 5 " indicates it is definitely true. A higher score indicates higher deficits and a lower score indicates lower deficits. The ADEXI was explicitly developed to investigate deficits in working memory and inhibition and address the limitations of other rating instruments that often include items overlapped with ADHD symptom levels. This instrument has proven to discriminate well between adults with ADHD and controls (Holst \& Thorell, 2018). Alpha for the total scale in current data is .91 and .90 for working memory deficits and .77 for inhibition deficits.

7) Existential Annihilation Anxiety (Kira et al., 2020b; Kira, Shuwiekh, Kucharska, \& Al-Huwailah, 2019). We used two subscales of the existential annihilation measure. One subscale measures existential annihilation anxiety related to identity status ( 4 items)and the second subscale measures existential anxiety related to death (3 items). Items scored on a four-point scale, with " 0 " indicates "completely disagree" and " 3 " indicates "completely agree". The measure and its subscales were validated in different languages including Arabic in previous studies. The two subscales have Cronbach alpha reliabilities of .834 for status existential annihilation anxiety subscale and .843 for death existential annihilation anxiety subscale in current data.

\section{Statistical Analysis}

We used IBM-SPSS 22 and Amos 22 software to analyze the data. The inspection of the variables indicated that there are no missing values in the data as the survey was designed such that it was not possible to proceed without entering a response to each item. To verify that trauma types I, II and III are different in their intensity regarding their effects on mental health and cognitive variables, we conducted a zero-order correlation between Type I, Type II, and Type III traumas and measures of PTSD, depression, generalized anxiety, existential status and death anxieties, and working memory and inhibition deficits. The goal was to compare the strength of association between the three trauma types and the mental health and executive function variables. To determine the significance of correlation size differences, we used Lee \& Preacher's (2013), software to calculate the fisher $\mathrm{z}$ score to compare correlations (see also Steiger, 1980).

We conducted path analysis, with the six trauma types (attachment, personal identity trauma, collective identity, physical identity survival, role identity, and secondary) as interacting independent variables, and PTSD, depression, generalized anxiety, working memory, and inhibition deficits as interacting outcome 
variables. Following Byrne's (2012) recommendations, the criteria for good model fit were a non-significant $\left(\chi^{2}\right),\left(\chi^{2} /\right.$ d.f. $\left.>2\right)$, comparative fit index (CFI) values $>0.90$, and root-mean-square error of approximation (RMSEA) values $<0.08$. However, we did not utilize $\mathrm{X}^{2}$ criteria as they are sensitive to large sample sizes, and our sample size $\mathrm{N}=2732$ is considered large (e.g., Meade \& Lautenschlager, 2004).

\section{Results}

1) Correlation results: All trauma types had significant correlations with PTSD, depression, anxiety, status and death existential anxieties, working memory and inhibition deficits, suicidality, and executive control deficits. Type II had higher associations with all variables than type I, and type III had higher associations with all variables than type II (and type I). The differences in association sizes between variables were significant. Table 1 presents the correlations between trauma types and the main variables, while Table 2 presents the $\mathrm{z}$ scores of the significance of differences in correlation sizes between trauma types.

2) Path analysis results: Attachment traumas, directly and indirectly, affected personal identity, collective identity, physical identity (survival), role identity, and secondary traumas. It had direct negative effects on generalized anxiety and PTSD; however, its indirect and total effects are positive and significant. It had indirect effects on depression, working memory, and inhibition deficits. Its direct effects on collective identity traumas accounted for $84 \%$ of its total effects. Its direct effects on personal identity trauma accounted for $43 \%$ of its total effects. Its direct effects on physical survival traumas accounted for $37 \%$ of its total

Table 1. Zero-order correlations between trauma types and mental health and exective functions variables.

\begin{tabular}{|c|c|c|c|c|c|c|c|c|c|c|c|c|}
\hline Variable & 1 & 2 & 3 & 4 & 5 & 6 & 7 & 8 & 9 & 10 & 11 & 12 \\
\hline 1) Type I Trauma & 1 & & & & & & & & & & & \\
\hline 2) Type II Trauma & $.76^{* * *}$ & 1 & & & & & & & & & & \\
\hline 3) Type III Trauma & $.69^{* * *}$ & $.88^{\star \star \star}$ & 1 & & & & & & & & & \\
\hline 4) PTSD & $.17^{\star * *}$ & $.20^{* * *}$ & $.28^{\star * *}$ & 1 & & & & & & & & \\
\hline 5) Depression & $.23^{* * *}$ & $.27^{\star * *}$ & $.34^{* * *}$ & $.71^{\star * *}$ & 1 & & & & & & & \\
\hline 6) Anxiety & $.16^{* * *}$ & $.19^{* * *}$ & $.26^{\star * *}$ & $.70^{\star * *}$ & $.78^{* * *}$ & 1 & & & & & & \\
\hline 7) Status EAA & $.20^{\star * *}$ & $.24^{\star * *}$ & $.29^{* * *}$ & $.45^{\star * *}$ & $.47^{* * *}$ & $.48^{* * *}$ & 1 & & & & & \\
\hline 8) Death EAA & $.16^{* * *}$ & $.21^{\star * *}$ & $.25^{\star * *}$ & $.50^{\star * \star}$ & $.51^{* * *}$ & $.54^{\star \star \star}$ & $.60^{* * *}$ & 1 & & & & \\
\hline $\begin{array}{l}\text { 9) Working } \\
\text { Memory Deficits }\end{array}$ & $.09^{* * *}$ & $.14^{\star * *}$ & $.19^{\star * *}$ & $.43^{\star * *}$ & $.38^{* * *}$ & $.34^{\star * *}$ & $.23^{\star * *}$ & $.28^{\star * *}$ & 1 & & & \\
\hline $\begin{array}{l}\text { 10) Inhibition } \\
\text { Deficits }\end{array}$ & $.17^{\star * *}$ & $.22^{* * *}$ & $.28^{* * *}$ & $.47^{\star * *}$ & $.42^{* * *}$ & $.38^{* * *}$ & $.23^{\star \star \star}$ & $.30^{* * *}$ & $.73^{* * *}$ & 1 & & \\
\hline 11) Suicidality & $.29^{* * *}$ & $.36^{* * *}$ & $.40^{* * *}$ & $.47^{\star * *}$ & $.53^{* * *}$ & $.47^{* * *}$ & $.44^{* * *}$ & $.50^{* * *}$ & $.250^{* *}$ & $.274^{\star *}$ & 1 & \\
\hline $\begin{array}{l}\text { 12) Executive } \\
\text { control deficit }\end{array}$ & $.20^{* * *}$ & $.25^{\star * *}$ & $.30^{* * *}$ & $.62^{\star * *}$ & $.65^{\star * *}$ & $.64^{\star * *}$ & $.421^{\star *}$ & $.50^{* * *}$ & $.45^{\star * *}$ & $.43^{* * *}$ & $.65^{\star * *}$ & 1 \\
\hline
\end{tabular}

Notes: ${ }^{*} p<.05,{ }^{* *} p<.01,{ }^{* *} p<.001$. 
Table 2. $\mathrm{z}$ scores for the significance of the difference in correlations size between types I, II, and III traumas and the main variables in the study.

\begin{tabular}{ccl}
\hline Variables & $\begin{array}{c}\text { The significance of the difference between } \\
\text { Type } 1 \text { and type II traumas }\end{array}$ & $\begin{array}{l}\text { The significance of the difference } \\
\text { between types II and III traumas }\end{array}$ \\
\hline PTSD & $\mathrm{z}$ score $=-2.31, \mathrm{p}($ two tails $)=.022$ & $\mathrm{z}$ score $=-8.82, \mathrm{p}($ two tails $)=.000$ \\
Depression & $\mathrm{z}$ score $=-3.01, \mathrm{p}($ two tails $)=.001$ & $\mathrm{z}$ score $=-7.88, \mathrm{p}($ two tails $)=.000$ \\
Generalized Anxiety & $\mathrm{z}$ score $=-2.30, \mathrm{p}($ two tails $)=.02$ & $\mathrm{z}$ score $=-7.69, \mathrm{p}($ two tails $)=.000$ \\
Status EAA & $\mathrm{z}$ score $=-3.10, \mathrm{p}($ two tails $)=.002$ & $\mathrm{z}$ score $=-5.55, \mathrm{p}($ two tails $)=.000$ \\
Death EAA & $\mathrm{z}$ score $=-3.85, \mathrm{p}($ two tails $)=.000$ & $\mathrm{z}$ score $=-4.39, \mathrm{p}($ two tails $)=.000$ \\
Working Memory Deficits & $\mathrm{z}$ score $=-3.80, \mathrm{p}($ two tails $)=.000$ & $\mathrm{z}$ score $=-5.41, \mathrm{p}($ two tails $)=.000$ \\
Inhibition Deficits & $\mathrm{z}$ score $=-3.86, \mathrm{p}($ two tails $)=.000$ & $\mathrm{z}$ score $=-6.63, \mathrm{p}($ two tails $)=.000$ \\
Suicidality & $\mathrm{z}$ score $=-5.63, \mathrm{p}($ two tails $)=.000$ & $\mathrm{z}$ score $=-4.64, \mathrm{p}($ two tails $)=.000$ \\
Executive control deficit & $\mathrm{z}$ score $=-3.88, \mathrm{p}($ two tails $)=.000$ & $\mathrm{z}$ score $=-6.13, \mathrm{p}($ two tails $)=.000$ \\
\hline
\end{tabular}

effects. Its direct effects on role identity traumas accounted for $40 \%$ of its total effects. Its direct effects on secondary traumas accounted for $19 \%$ of its total effects. No other traumas affected attachment traumas directly or indirectly, which makes more sense as attachment traumas are the earliest in life and proliferate to all other subsequent traumas. Its total effect size on the other subsequent traumas ranged from high $(.74, .73$, and .72 for personal identity, collective and secondary traumas) to moderate (.57 for role identity traumas). Its total effect size on depression, PTSD, working memory, and inhibition deficits was small but highly significant.

Personal identity traumas had direct and indirect effects on physical identity (survival) traumas, role identity traumas and secondary traumas, and PTSD. It had indirect effects on itself (loop), cumulative identity traumas, depression, working memory, and inhibition deficits. Its direct effects on physical survival traumas accounted for .88 of its total effects. Its direct effects on role identity (achievement/status) trauma accounted for .73 of its total effects. Its direct effects on secondary traumas accounted for .45 of its total effects. Its direct effects on role generalized anxiety accounted for .80 of its total effects. Its direct effects on secondary traumas accounted for .45 of its total effects. Its direct effects on PTSD accounted for .73 of its total effects. Its direct effects on secondary traumas accounted for .21 of its total effects. Its total effect sizes on physical survival traumas (.51), secondary traumas (.47), and role identity trauma (.40) were moderate. Its total effects on collective identity trauma (.09) were small but significant. Its total effect sizes on generalized anxiety (.35), PTSD (.34), depression (.31) were moderate to low but highly significant. Its total effects on inhibition deficits (23) and working memory deficits (18) were low but significant.

Collective identity trauma, directly and indirectly, affected personal identity traumas, physical identity (survival) traumas, secondary traumas, and depression. It had indirect effects on itself (loop) and indirect effects on role identity traumas, generalized anxiety, PTSD, working memory, and inhibition deficits. 
Its direct effects on personal identity traumas accounted for .95 of its total effects. Its direct effects on physical identity (survival) traumas accounted for .27 of its total effects. Its direct effects on secondary traumas accounted for .33 of its total effects. Its direct effects on depression accounted for .24 of its total effects. Its total effect sizes on personal identity trauma (.60) were high to moderate. Its total effect sizes on the other trauma types were moderate to low. Its total effects on depression, generalized anxiety, PTSD, inhibition, and working memory deficits were on the low side, but all were highly significant.

Physical survival trauma had direct and indirect effects on role identity and secondary traumas. It had indirect effects on all the other trauma types (except attachment). It had indirect effects on PTSD, depression, generalized anxiety, working memory, and inhibition deficits. Its direct effects on role (status) identity traumas accounted for .95 of its total effects. Its direct effects on secondary traumas accounted for .91 of its total effects. While its total effect size on secondary traumas is moderate (.42), Its effect sizes on the other traumas and mental health and executive functions are relatively small but significant.

Role identity (achievement) traumas affected directly and indirectly collective identity traumas, secondary traumas, generalized anxiety, depression, PTSD, working memory, and inhibition deficits. Its direct effects on collective identity traumas accounted for .96 of its total effects. Its direct effects on secondary traumas accounted for .50 of its total effects. Its direct effects on working memory deficits accounted for .47 of its total effects. Its direct effects on inhibition deficits accounted for .20 of its total effects. All of its total effects were relatively small but significant.

Secondary traumas had direct effects on depression, direct and indirect effects on inhibition deficits, and indirect effects on working memory and PTSD. Its direct effects on inhibition deficits accounted for .82 of its total effects. Its total effects in all the affected variables were relatively small and significant.

Generalized anxiety had a direct effect on depression, direct and indirect effects on PTSD and indirect effects on working memory and inhibition deficits. Its direct effects on PTSD account for 55\% of its total effects. Its total effects on depression and PTSD are large. Its total effects on working memory and inhibition deficits were medium to small. Depression had direct medium-size effects on PTSD, direct and indirect (medium to small size) effects on working memory, and inhibition deficits. PTSD had direct effects (medium to small) on working memory and direct and indirect effects on inhibition deficits.

We should note that the only traumas that directly affected executive function deficits in this model were role identity and secondary traumas with small effect sizes. However, the direct impact with moderate effect sizes was from depression and PTSD. All other trauma times had an indirect impact on executive functions that ranged from small to medium. Table 3 presents the direct, indirect and total effects and .95 confidence intervals for each variable in the model. Figure 1 presents the path diagram of the model with all the direct paths between variables. 
Table 3. The direct, indirect, and total effects and their 95\% confidence intervals of each variable in the model.

\begin{tabular}{|c|c|c|c|c|c|c|c|c|c|c|}
\hline \multirow[b]{2}{*}{$\begin{array}{c}\text { Causal } \\
\text { Variables }\end{array}$} & \multicolumn{10}{|c|}{ Endogenous Variables } \\
\hline & PIT & CIT & PST & RIT & $\begin{array}{l}\text { General } \\
\text { Anxiety }\end{array}$ & ST & Dep. & PTSD & $\begin{array}{c}\text { Working } \\
\text { Memory } \\
\text { D. }\end{array}$ & $\begin{array}{l}\text { Inhibition } \\
\text { Deficits }\end{array}$ \\
\hline \multicolumn{11}{|c|}{ Attachment traumas } \\
\hline $\begin{array}{l}\text { Direct } \\
\text { Effects }\end{array}$ & $\begin{array}{c}.32^{\star *} \\
(.26 / .37)\end{array}$ & $\begin{array}{c}.61^{\star *} \\
(.57 / .65)\end{array}$ & $\begin{array}{c}.25^{\star *} \\
(.18 / .30)\end{array}$ & $\begin{array}{c}.23^{\star *} \\
(.17 / .29)\end{array}$ & $\begin{array}{c}-.15^{\star *} \\
(-.23 /-.09)\end{array}$ & $\begin{array}{c}.14^{*} \\
(.09 / .18)\end{array}$ & & $\begin{array}{c}-.07^{\star \star} \\
(-.11 /-.03)\end{array}$ & & \\
\hline $\begin{array}{l}\text { Indirect } \\
\text { Effects }\end{array}$ & $\begin{array}{c}.42^{\star *} \\
(.37 / .46)\end{array}$ & $\begin{array}{c}.12^{\star *} \\
(.09 / .15)\end{array}$ & $\begin{array}{c}.43^{* * *} \\
(.39 / .46)\end{array}$ & $\begin{array}{c}.34^{\star *} \\
(.29 / .40)\end{array}$ & $\begin{array}{c}.29^{* *} \\
(.23 / .34)\end{array}$ & $\begin{array}{c}.58^{\star \star} \\
(.54 / .61)\end{array}$ & $\begin{array}{c}.21^{\star *} \\
(.16 / .25)\end{array}$ & $\begin{array}{c}.21^{\star *} \\
(.16 / .24)\end{array}$ & $\begin{array}{c}11^{* *} \\
(.09 / .14)\end{array}$ & $\begin{array}{l}.18^{\star * *} \\
(.16 / .21)\end{array}$ \\
\hline Total Effects & $\begin{array}{c}.74^{\star *} \\
(.71 / .77)\end{array}$ & $\begin{array}{c}.73^{* *} \\
(.70 / .77)\end{array}$ & $\begin{array}{c}.68^{* *} \\
(.64 / .71)\end{array}$ & $\begin{array}{c}.57^{* *} \\
(.53 / .61)\end{array}$ & $\begin{array}{c}.13^{* *} \\
(.08 / .18)\end{array}$ & $\begin{array}{c}.72^{\star *} \\
(.69 / .74)\end{array}$ & $\begin{array}{c}.21^{\star *} \\
(.16 / .25)\end{array}$ & $\begin{array}{c}.14^{\star *} \\
(.09 / .17)\end{array}$ & $\begin{array}{c}11^{* *} \\
(.09 / .14)\end{array}$ & $\begin{array}{c}.18^{* * *} \\
(.16 / .21)\end{array}$ \\
\hline
\end{tabular}

\section{Personal identity traumas}

\begin{tabular}{|c|c|c|c|c|c|c|c|c|c|c|}
\hline $\begin{array}{l}\text { Direct } \\
\text { Effects }\end{array}$ & & & $\begin{array}{c}.45^{\star *} \\
(.42 / .54)\end{array}$ & $\begin{array}{c}.29^{\star \star} \\
(.21 / .35)\end{array}$ & $\begin{array}{c}.28^{\star \star} \\
(.20 / .36)\end{array}$ & $\begin{array}{c}.21^{\star *} \\
(.17 / .26)\end{array}$ & & $\begin{array}{l}.07^{\star * \star} \\
(.04 / .11)\end{array}$ & & \\
\hline $\begin{array}{l}\text { Indirect } \\
\text { Effects }\end{array}$ & $\begin{array}{c}.05^{\star *} \\
(.04 / .06)\end{array}$ & $\begin{array}{c}.09^{* *} \\
(.07 / .11)\end{array}$ & $\begin{array}{c}.06^{* * *} \\
(.04 / .06)\end{array}$ & $\begin{array}{c}.11^{\star *} \\
(.08 / 14)\end{array}$ & $\begin{array}{c}.07^{\star *} \\
(.05 / .09)\end{array}$ & $\begin{array}{c}.26^{* *} \\
(.22 / .29)\end{array}$ & $\begin{array}{c}.31^{* *} \\
(.26 / .36)\end{array}$ & $\begin{array}{c}.27^{\star *} \\
(.22 / .32)\end{array}$ & $\begin{array}{c}.18^{* * *} \\
(.16 / .22)\end{array}$ & $\begin{array}{c}.23^{* * *} \\
(.20 / .25)\end{array}$ \\
\hline Total Effects & $\begin{array}{c}.05^{\star *} \\
(.04 / .06)\end{array}$ & $\begin{array}{c}.09^{\star *} \\
(.07 / .11)\end{array}$ & $\begin{array}{c}.51^{\star *} \\
(.46 / .59)\end{array}$ & $\begin{array}{c}.40^{* \star} \\
(.33 / .46)\end{array}$ & $\begin{array}{c}.35^{\star *} \\
(.29 / .42\end{array}$ & $\begin{array}{c}.47^{\star} \\
(.41 / .51)\end{array}$ & $\begin{array}{c}.31^{\star *} \\
(.26 / .36)\end{array}$ & $\begin{array}{c}.34^{\star *} \\
(.29 / .41)\end{array}$ & $\begin{array}{c}.18^{\star * *} \\
(.16 / .22)\end{array}$ & $\begin{array}{c}.23^{* * *} \\
(.20 / .25)\end{array}$ \\
\hline \multicolumn{11}{|c|}{ Collective identity traumas } \\
\hline $\begin{array}{l}\text { Direct } \\
\text { Effects }\end{array}$ & $\begin{array}{c}.57^{\star *} \\
(.52 / .62)\end{array}$ & 工 & $\begin{array}{c}.10^{* *} \\
(.04 / .15)\end{array}$ & & 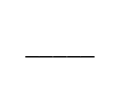 & $\begin{array}{c}.15^{* *} \\
(.10 / .19)\end{array}$ & $\begin{array}{c}.06^{* *} \\
(.03 / .09)\end{array}$ & & & \\
\hline $\begin{array}{l}\text { Indirect } \\
\text { Effects }\end{array}$ & $\begin{array}{c}.03^{\star *} \\
(.02 / .04)\end{array}$ & $\begin{array}{c}.05^{\star \star \star} \\
(.04 / .07)\end{array}$ & $\begin{array}{c}.29^{\star *} \\
(.25 / .33)\end{array}$ & $\begin{array}{c}.25^{\star *} \\
(.20 / .28)\end{array}$ & $\begin{array}{c}.20^{\star * *} \\
(.17 / .24)\end{array}$ & $\begin{array}{c}.31^{\star *} \\
(.25 / .34)\end{array}$ & $\begin{array}{c}.19^{* *} \\
(.16 / .23)\end{array}$ & $\begin{array}{c}.23^{* * *} \\
(.20 / .26)\end{array}$ & $\begin{array}{c}.12^{\star *} \\
(.10 / .14)\end{array}$ & $\begin{array}{c}.17^{* *} \\
(.15 / .19)\end{array}$ \\
\hline Total Effects & $\begin{array}{c}.60^{\star *} \\
(.55 / .66)\end{array}$ & $\begin{array}{c}.05^{\star * *} \\
(.04 / .07)\end{array}$ & $\begin{array}{c}.39^{* *} \\
(.33 / .44)\end{array}$ & $\begin{array}{c}.25^{\star *} \\
(.20 / .28)\end{array}$ & $\begin{array}{l}.20^{\star * \star} \\
(.17 / .24)\end{array}$ & $\begin{array}{c}.46^{* \star} \\
(.41 / .51)\end{array}$ & $\begin{array}{c}.25^{\star *} \\
(.21 / .30)\end{array}$ & $\begin{array}{l}.23^{* * *} \\
(.20 / .26)\end{array}$ & $\begin{array}{c}.12^{\star \star} \\
(.10 / .14)\end{array}$ & $\begin{array}{c}.17^{\star *} \\
(.15 / .19)\end{array}$ \\
\hline
\end{tabular}

Physical survival traumas

\begin{tabular}{|c|c|c|c|c|c|c|c|c|c|c|}
\hline Direct & & & & $.19^{* *}$ & & $.38^{\star}$ & & & & \\
\hline Effects & & & & $(.14 / .23)$ & & $(.34 / .41)$ & & & & \\
\hline $\begin{array}{l}\text { Indirect } \\
\text { Effects }\end{array}$ & $\begin{array}{c}.02^{* *} \\
(.02 / .03)\end{array}$ & $\begin{array}{c}.04^{* *} \\
(.03 / .06)\end{array}$ & $\begin{array}{c}.02^{* *} \\
(.01 / .02)\end{array}$ & $\begin{array}{c}.01^{\star *} \\
(.01 / .01)\end{array}$ & $\begin{array}{c}.04^{\star *} \\
(.02 / .05)\end{array}$ & $\begin{array}{c}.04^{* *} \\
(.02 / .05)\end{array}$ & $\begin{array}{c}.06^{* *} \\
(.04 / .08)\end{array}$ & $\begin{array}{c}.05^{\star *} \\
(.04 / .06)\end{array}$ & $\begin{array}{c}.04^{* *} \\
(.03 / .05)\end{array}$ & $\begin{array}{c}.08^{* *} \\
(.06 / .09)\end{array}$ \\
\hline Total Effects & $\begin{array}{c}.02^{\star *} \\
(.02 / .03)\end{array}$ & $\begin{array}{c}.04^{\star *} \\
(.03 / .06)\end{array}$ & $\begin{array}{c}.02^{* *} \\
(.01 / .02)\end{array}$ & $\begin{array}{c}.20^{* *} \\
(.15 / .24)\end{array}$ & $\begin{array}{c}.04^{* *} \\
(.02 / .05)\end{array}$ & $\begin{array}{c}.42^{\star} \\
(.37 / .45)\end{array}$ & $\begin{array}{c}.06^{* *} \\
(.04 / .08)\end{array}$ & $\begin{array}{c}.05^{\star *} \\
(.04 / .06)\end{array}$ & $\begin{array}{c}.04^{* *} \\
(.03 / .05)\end{array}$ & $\begin{array}{c}.08^{* *} \\
(.06 / .09)\end{array}$ \\
\hline Role identity & raumas & & & & & & & & & \\
\hline $\begin{array}{l}\text { Direct } \\
\text { Effects }\end{array}$ & & $\begin{array}{c}.21^{\star *} \\
(.16 / .26)\end{array}$ & & & $\begin{array}{c}.14^{\star *} \\
(.09 / .18)\end{array}$ & $\begin{array}{c}.10^{* *} \\
(.07 / .15)\end{array}$ & $\begin{array}{c}.04 \\
(.00 / .08)\end{array}$ & $\begin{array}{c}.04 \\
(-.00 / .07)\end{array}$ & $\begin{array}{c}.08^{\star *} \\
(.04 / .11)\end{array}$ & $\begin{array}{c}.04^{*} \\
(.00 / .06)\end{array}$ \\
\hline $\begin{array}{l}\text { Indirect } \\
\text { Effects }\end{array}$ & $\begin{array}{c}.13^{\star *} \\
(.09 / .16)\end{array}$ & $\begin{array}{c}.01^{* *} \\
(.01 / .02)\end{array}$ & $\begin{array}{c}.08^{* *} \\
(.06 / .10)\end{array}$ & $\begin{array}{c}.05^{\star * *} \\
(.04 / .06)\end{array}$ & $\begin{array}{c}.04^{\star *} \\
(.03 .06) /\end{array}$ & $\begin{array}{c}.10^{\star *} \\
(.06 / .12)\end{array}$ & $\begin{array}{c}.17^{\star \star} \\
(.12 / .20)\end{array}$ & $\begin{array}{c}.16^{\star *} \\
(.12 / .19)\end{array}$ & $\begin{array}{c}.09^{* *} \\
(.07 / .11)\end{array}$ & $\begin{array}{c}.16^{\star *} \\
(.13 / .19)\end{array}$ \\
\hline Total Effects & $\begin{array}{c}.13^{* *} \\
(.09 / .16)\end{array}$ & $\begin{array}{c}.22^{* *} \\
(.17 / .28)\end{array}$ & $\begin{array}{c}.08^{* *} \\
(.06 / .10)\end{array}$ & $\begin{array}{c}.05^{* * *} \\
(.04 / .06)\end{array}$ & $\begin{array}{c}.18^{* *} \\
(.13 / .22)\end{array}$ & $\begin{array}{c}.20^{* *} \\
(.16 / .23)\end{array}$ & $\begin{array}{c}.21^{\star *} \\
(.15 / .25)\end{array}$ & $\begin{array}{c}.20^{\star \star} \\
(.15 / .25)\end{array}$ & $\begin{array}{c}.17^{\star \star} \\
(.13 / .20)\end{array}$ & $\begin{array}{c}.20^{* *} \\
(.16 / .24)\end{array}$ \\
\hline
\end{tabular}




\section{Continued}

\section{Generalized anxiety}

Direct

Effects

Indirect

Effects
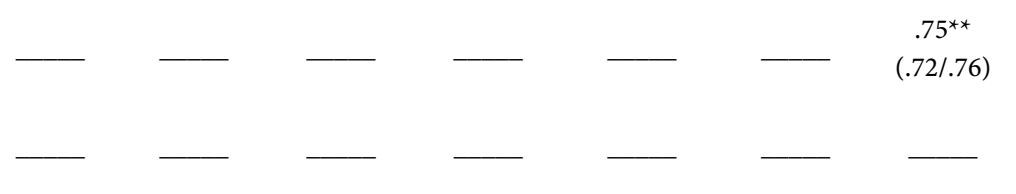

$.36^{* *}$

$(.72 / .76) \quad(.32 / .41)$

Total Effects

\section{Secondary Traumas}

Direct

Effects

Indirect

Effects

Total Effects
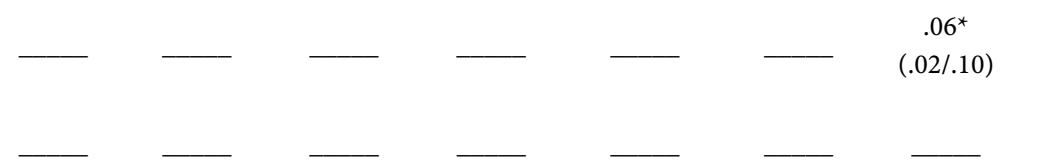

$\begin{array}{ccc}.30^{* *} & .31^{\star *} & .32^{\star *} \\ (.26 / .33) & (.28 / .33) & (.30 / .35) \\ & & \\ .66^{* *} & .31^{\star *} & .32^{\star *} \\ (.64 / .63) & (.28 / .33) & (.30 / .35)\end{array}$

$.75^{* *}$

$(.72 / .76)$

$(.64 / .63)$

$(.28 / .33)$

$(.30 / .35)$

\section{Depression}

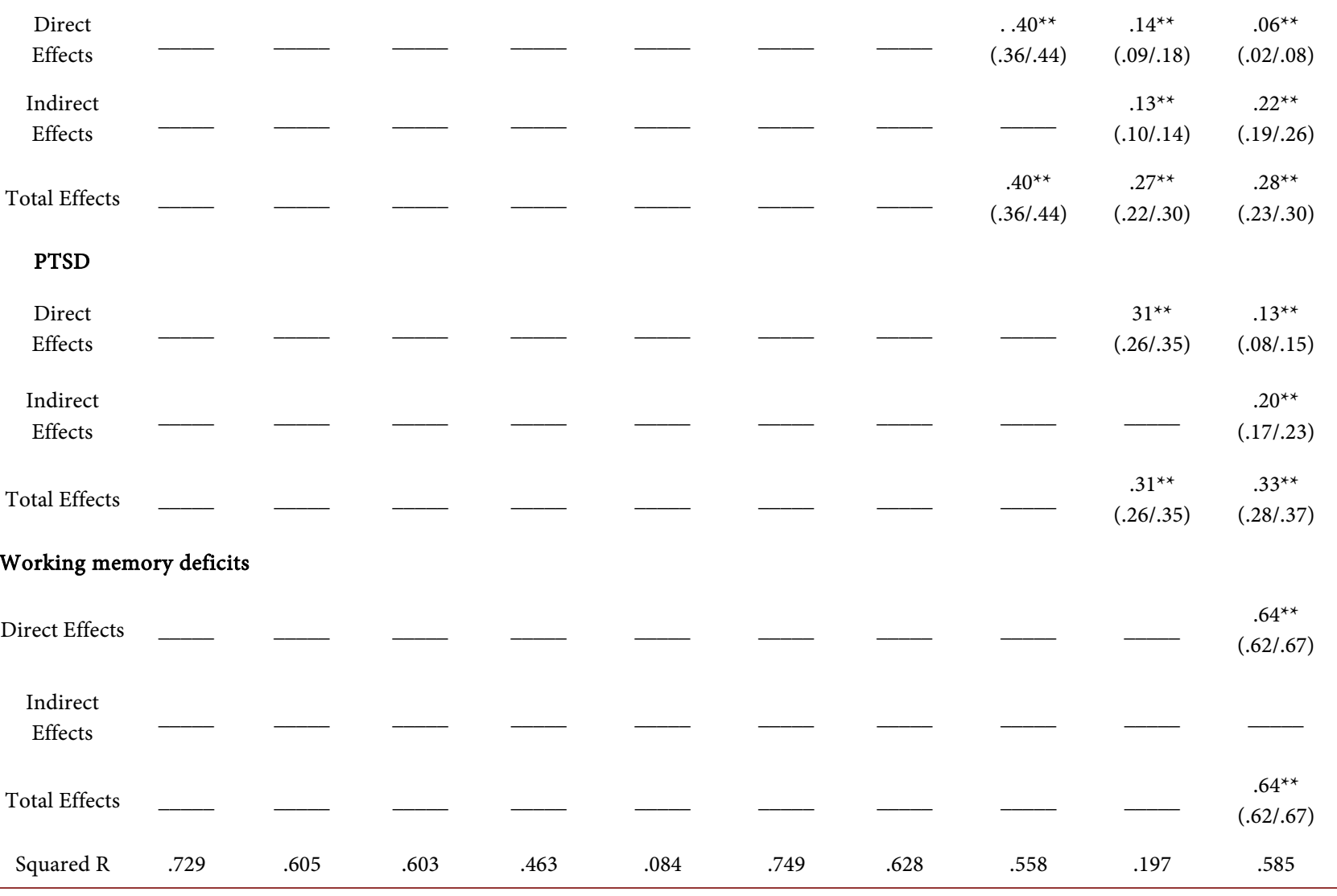

Notes: ${ }^{*} p<.05,{ }^{*} p<.01,{ }^{* *} p<.001$. Notes: $\mathrm{PIT}=$ personal identity traumas; $\mathrm{CIT}=$ Collective identity traumas; $\mathrm{PST}=$ Physical survival traumas RIT $=$ Role identity traumas; $\mathrm{ST}=$ Secondary traumas; Dep. $=$ Depression.

\section{Discussion}

The study provided evidence for the validity of some of the main parameters of DBTF. It is the first study that provided empirical evidence of the types I, II, and III traumas as a measure of the gradient impact severity of each trauma type, 
$\mathrm{N}=2732-11$ Arab countries

Chi Square $=66.445$, d.f. $=20, p=.000$

$\mathrm{CFI}=.998$

RMSEA $=.029$

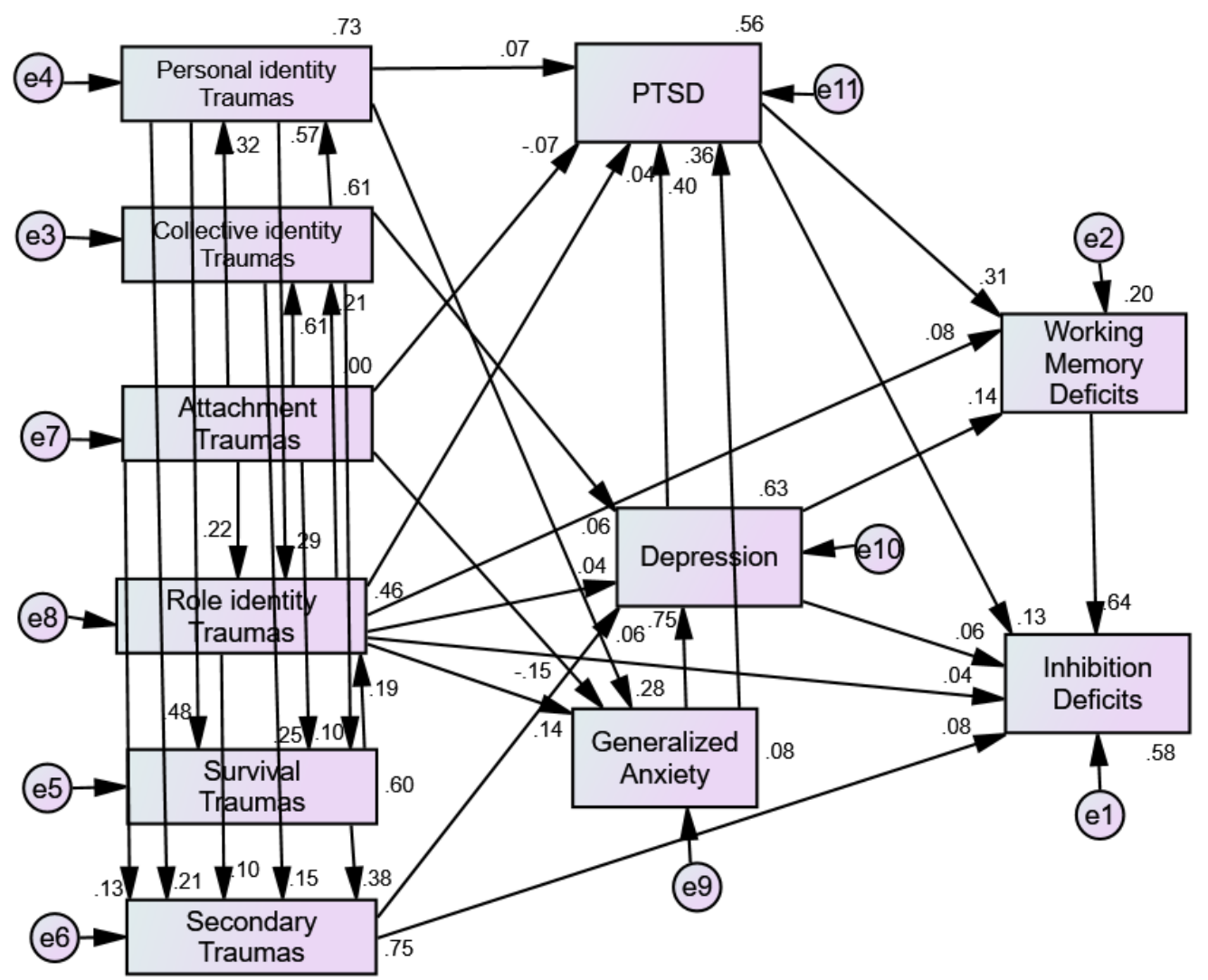

Figure 1. Path diagram with all the direct paths between variables in the model.

with type III trauma, e.g., discrimination, has the worst impact on mental health and executive functions compared to types I and II. The results replicated and extended previous findings on the impact of discrimination as the worse trauma types considering its impact on minorities (Kira et al., 2008b). Discrimination is a continuous life trauma contributing to persistent disparities in morbidity and mortality rates among minority groups. Additionally, the study provided further evidence for the proliferation of early traumas, with attachment, personal identity (early childhood adversities), and collective identity traumas leading the proliferation to other trauma dynamics. That replicated and extended previous findings on trauma and stress proliferation dynamics (Kira et al., 2018a; Pearlin et al., 1997). Also, attachment traumas have a larger effect size in the trauma proliferation dynamics. However, while attachment traumas have the largest effect size on other traumas, personal identity trauma and collective identity trauma had the highest impact on mental health and cognitive functioning. The signifi- 
cant impact of attachment trauma on mental health and cognitive functioning is primarily indirect, mediated by its high impact on other trauma types.

The study results have significant conceptual, stress and trauma measurement, and clinical implications. Conceptually, the potentials of DBTF in transforming the landscape of stress and trauma research are yet to be fully harnessed. We advance various assertions regarding the nature of stress and trauma from a developmental perspective. First, the single trauma-focused approach on conceptualizing and treating traumatization outcomes may be missing the mark of the whole picture and the powerful dynamics of traumatization that drive the interaction of stressors (acute and non-acute), individual development, and behavior. Interacting stressors and traumas factors and processes matter for the developing individual till the end of life. Second, the intensity of stressors (acute, traumatic, or chronic) is essential in predicting their outcome, with continuous stressors (traumatic and chronic) (type III) such as discrimination and early childhood adversities being the worse regarding their impact on mental health and cognitive functioning. Third, in real life, poly-traumatization, severe trauma, and cumulative trauma, in multiply severely traumatized rarely present its outcome in a single diagnosis, but rather in a syndrome or profile of comorbidity, including PTSD, complex PTSD, depression, anxiety, suicidality, and executive function deficits. We should instead identify profiled syndromes to address them more effectively in treatment. Finally, the focus on mortality salience and physical traumatization alone, ignoring the existence's salience of the developing identity with its complex structure and dynamics that exist in time and space, is not a tenable position that allows us to understand the phenomena of traumatization in real-time and real-life and its impact on minorities and non-minority populations. It is time to re-focus and reconceptualize the field of stress and trauma around the person's development with diverging salience of both her/his developing existence (identity) and mortality.

From the stressors and traumas measurement perspective, while not primarily intended in the study design, the current study provided strong evidence that cumulative stressors and traumas measure has robust psychometrics and provided evidence of its construct and predictive validity of all its primary measured constructs. The measure was initially developed (22 and 32 items) based on the DBTF framework (Kira et al., 2008a). The furtherly developed version we used in the study is currently 36 items with more representative items of the basic constructs of DBTF (the current version is attached as Appendix 1).

Clinically, the results have significant implications for precision intervention and prevention. Preventive social interventions that prevent or minimize attachment and early childhood traumas and interventions that suppress early on attachment and childhood traumas proliferation may be helpful. Attachment trauma-focused, early childhood trauma-focused, identity-focused, and continuous trauma-focused (type III traumas) interventions may be developed to supplement the current evidence-based packages. The current single trauma-focused 
intervention seems to have limited efficacy. For example, a meta-analysis concluded that recommended interventions appear less effective at relatively low and high patient PTSD severity levels (Haagen et al., 2015). Current intensive interventions to treat and prevent suicide did not stop the spiral escalation of suicide rates (Goldston et al., 2015; Luby \& Kertz, 2019). Identity-focused and continuous trauma-focused interventions (e.g., Eichas et al., 2015; Kira et al., 2015a; Umaña-Taylor \& Douglass, 2017) proved helpful. Attachment-focused interventions need to be further developed (e.g., Berlin, 2005; Steele \& Steele, 2017). Considering that executive function deficits were a significant outcome of different trauma types and their associated mental health syndromes, any treatment package may include cognitive training and other intervention that enhance executive functions. Cognitive training (for meta-analysis: Jahn et al., 2021) and acute exercise training (e.g., Pontifex et al., 2021) that enhance executive functions can be some of the keys to their treatment. Research into cognitive functions across psychological disorders suggests that multiple disorders may present cognitive deficiencies, potentially pointing to a transdiagnostic phenomenon (Abramovitch et al., 2021).

The current study had various limitations. The study was conducted in a convenient sample using a cross-sectional design. Cross-sectional design can report an association with no conclusions on cause and effects that only longitudinal design can achieve. Further, the measures used are based on participants' selfreports. Self-reports are subject to under-or over-reporting due to social desirability. We used a self-report measure, not a performance measure, to test for executive functions. However, previous research has demonstrated that task-based and self-report measures of EF capture complementary yet distinct components of cognitive control (Snyder et al., 2020). Further, we must caution that the direct and indirect effects used in path analysis do not mean the same thing in the deterministic sciences of cause and effect. Regardless, the study provided evidence that demonstrated the dynamics of intersected discrimination on COVID19 infection and stressors in minorities.

\section{Conflicts of Interest}

The author declares no conflicts of interest regarding the publication of this paper.

\section{References}

Abdou, R., Cassells, D., Berrill, J., \& Hanly, J. (2020). An Empirical Investigation of the Relationship between Business Performance and Suicide in the US. Social Science \& Medicine, 264, 113256.

Abramovitch, A., Short, T., \& Schweiger, A. (2021). The c Factor: Cognitive Dysfunction as a Transdiagnostic Dimension in Psychopathology. Clinical Psychology Review, 86, Article ID: 102007. https://doi.org/10.1016/j.cpr.2021.102007

Abu Ras, W., Suárez, Z. E., \& Breiwish, R. R. (2021). Beyond the Axes of Inequality: Religion, Race, and Everything in between. American Journal of Orthopsychiatry, 91, 217- 


\section{5. https://doi.org/10.1037/ort0000478}

Alexander, J. C., Eyerman, R., Giesen, B., Smelser, N. J., \& Sztompka, P. (2004). Cultural Trauma and Collective Identity. University of California Press. https://doi.org/10.1525/california/9780520235946.001.0001

Ali, D. A., Figley, C. R., Tedeschi, R. G., Galarneau, D., \& Amara, S. (2021). Shared Trauma, Resilience, and Growth: A Roadmap toward Transcultural Conceptualization. Psychological Trauma: Theory, Research, Practice, and Policy. https://doi.org/10.1037/tra0001044

American Psychiatric Association, APA (2013). Diagnostic and Statistical Manual of Mental Disorders: DSM-5. Arlington, VA.

Ashmore, R. D., Deaux, K., \& McLaughlin-Volpe, T. (2004). An Organizing Framework for Collective Identity: Articulation and Significance of Multidimensionality. Psychological Bulletin, 130, 80. https://doi.org/10.1037/0033-2909.130.1.80

Bardol, O., Grot, S., Oh, H., Poulet, E., Zeroug-Vial, H., Brunelin, J., \& Leaune, E. (2020). Perceived Ethnic Discrimination as a Risk Factor for Psychotic Symptoms: A Systematic Review and Meta-Analysis. Psychological Medicine, 50, 1077-1089. https://doi.org/10.1017/S003329172000094X

Barnes, L. L., de Leon, C. F., Lewis, T. T., Bienias, J. L., Wilson, R. S., \& Evans, D. A. (2008). Perceived Discrimination and Mortality in a Population-Based Study of Older Adults. American Journal of Public Health, 98, 1241-1247. https://doi.org/10.2105/AJPH.2007.114397

Barnum, E. L., \& Perrone-McGovern, K. M. (2017). Attachment, Self-Esteem and Subjective Well-Being among Survivors of Childhood Sexual Trauma. Journal of Mental Health Counseling, 39, 39-55. https://doi.org/10.17744/mehc.39.1.04

Baum, N., Rahav, G., \& Sharon, M. (2014). Heightened Susceptibility to Secondary Traumatization: A Meta-Analysis of Gender Differences. American Journal of Orthopsychiatry, 84, 111-122. https://doi.org/10.1037/h0099383

Berlin, L. J. (2005). Interventions to Enhance Early Attachments: The State of the Field Today. In L. J. Berlin, Y. Ziv, L. Amaya-Jackson, \& M. T. Greenberg (Eds.), Enhancing Early Attachments: Theory, Research, Intervention, and Policy (pp. 3-33). Guilford Press.

Bowlby, J. (1979). The Bowlby-Ainsworth Attachment Theory. Behavioral and Brain Sciences, 2, 637-638. https://doi.org/10.1017/S0140525X00064955

Breslau, N., Chilcoat, H. D., Kessler, R. C., Peterson, E. L., \& Lucia, V. C.(1999). Vulnerability to Assaultive Violence: Further Specification of the Sex Difference in Post-Traumatic Stress Disorder. Psychological Medicine, 29, 813-821. https://doi.org/10.1017/S0033291799008612

Byrne, B. M. (2012). Choosing Structural Equation Modeling Computer Software: Snapshots of LISREL, EQS, AMOS, and Mplus. In R. H. Hoyle (Ed.), Handbook of Structural Equation Modeling (pp. 307-324). The Guilford Press.

Castellví, P., Miranda-Mendizábal, A., Alayo, I., Parés-Badell, O., Almenara, J., Alonso, I., Alonso, J. et al. (2020). Assessing the Relationship between School Failure and Suicidal Behavior in Adolescents and Young Adults: A Systematic Review and Meta-Analysis of Longitudinal Studies. School Mental Health, 12, 429-441. https://doi.org/10.1007/s12310-020-09363-0

Darling Rasmussen, P., Storebø, O. J., Løkkeholt, T., Voss, L. G., Shmueli-Goetz, Y., Bojesen, A. B., Bilenberg, N. et al. (2019). Attachment as a Core Feature of Resilience: A Systematic Review and Meta-Analysis. Psychological Reports, 122, 1259-1296. https://doi.org/10.1177/0033294118785577

de Freitas, D. F., Fernandes-Jesus, M., Ferreira, P. D., Coimbra, S., Teixeira, P. M., de 
Moura, A., Gato, J., Marques, S. C., \& Fontaine, A. M. (2018). Psychological Correlates of Perceived Ethnic Discrimination in Europe: A Meta-Analysis. Psychology of Violence, 8, 712-725. https://doi.org/10.1037/vio0000215

Díaz-Venegas, C., Downer, B., Langa, K. M., \& Wong, R. (2016). Racial and Ethnic Differences in Cognitive Function among Older Adults in the USA. International Journal of Geriatric Psychiatry, 31, 1004-1012. https://doi.org/10.1002/gps.4410

Dolezsar, C. M., McGrath, J. J., Herzig, A. J. M., \& Miller, S. B. (2014). Perceived Racial Discrimination and Hypertension: A Comprehensive Systematic Review. Health Psychology, 33, 20-34. https://doi.org/10.1037/a0033718

Eichas, K., Meca, A., Montgomery, M. J., \& Kurtines, W. M. (2015). Identity and Positive Youth Development: Advances in Developmental Intervention Science. In M. Syed, \& K. C. McLean (Eds.), The Oxford Handbook of Identity Development (pp. 337-354). Oxford University Press.

Epstein, S., Roberts, E., Sedgwick, R., Polling, C., Finning, K., Ford, T., Downs, J. et al. (2020). School Absenteeism as a Risk Factor for Self-Harm and Suicidal Ideation in Children and Adolescents: A Systematic Review and Meta-Analysis. European Child \& Adolescent Psychiatry, 29, 1175-1194. https://doi.org/10.1007/s00787-019-01327-3

Everson-Rose, S. A., Lutsey, P. L., Roetker, N. S., Lewis, T. T., Kershaw, K. N., Alonso, A., \& Diez Roux, A. V. (2015). Perceived Discrimination and Incident Cardiovascular Events: The Multi-Ethnic Study of Atherosclerosis. American Journal of Epidemiology, 182, 225-234. https://doi.org/10.1093/aje/kwv035

Figley, C. R. (1995). Compassion Fatigue: Coping with Secondary Traumatic Stress Disorder in Those Who Treat the Traumatized. Brummer/Mazel.

Gale, M. M., Pieterse, A. L., Lee, D. L., Huynh, K., Powell, S., \& Kirkinis, K. (2020). A Meta-Analysis of the Relationship between Internalized Racial Oppression and HealthRelated Outcomes. The Counseling Psychologist, 48, 498-525. https://doi.org/10.1177/0011000020904454

Goldston, D. B., Daniel, S. S., Erkanli, A., Heilbron, N., Doyle, O., Weller, B., Faulkner, M. et al. (2015). Suicide Attempts in a Longitudinal Sample of Adolescents Followed through Adulthood: Evidence of Escalation. Journal of Consulting and Clinical Psychology, 83, 253. https://doi.org/10.1037/a0038657

Haagen, J. F., Smid, G. E., Knipscheer, J. W., \& Kleber, R. J. (2015). The Efficacy of Recommended Treatments for Veterans with PTSD: A Metaregression Analysis. Clinical Psychology Review, 40, 184-194. https://doi.org/10.1016/j.cpr.2015.06.008

Haw, C., Hawton, K., Gunnell, D., \& Platt, S. (2015). Economic Recession and Suicidal Behaviour: Possible Mechanisms and Ameliorating Factors. International Journal of Social Psychiatry, 61, 73-81. https://doi.org/10.1177/0020764014536545

Hirschberger, G. (2018). Collective Trauma and the Social Construction of Meaning. Frontiers in Psychology, 9, 1441. https://doi.org/10.3389/fpsyg.2018.01441

Holst, Y., \& Thorell, L. B. (2018). Adult Executive Functioning Inventory (ADEXI): Validity, Reliability, and Relations to ADHD. International Journal of Methods in Psychiatric Research, 27, e1567.

Humphreys, K. L., LeMoult, J., Wear, J. G., Piersiak, H. A., Lee, A., \& Gotlib, I. H. (2020). Child Maltreatment and Depression: A Meta-Analysis of Studies Using the Childhood Trauma Questionnaire. Child Abuse \& Neglect, 102, Article ID: 104361. https://doi.org/10.1016/j.chiabu.2020.104361

Ibrahim, H., Ertl, V., Catani, C., Ismail, A. A., \& Neuner, F. (2018). The Validity of Posttraumatic Stress Disorder Checklist for DSM-5 (PCL-5) as Screening Instrument with Kurdish and Arab Displaced Populations Living in the Kurdistan Region of Iraq. BMC 
Psychiatry, 18, 1-8.

Inzlicht, M., Tullett, A. M., \& Gutsell, J. N. (2012). Stereotype Threat Spillover: The Short- and Long-Term Effects of Coping with Threats to Social Identity. In M. Inzlicht, \& T. Schmader (Eds.), Stereotype Threat: Theory, Process, and Application (pp. 107123). Oxford University Press. https://doi.org/10.1093/acprof:oso/9780199732449.003.0007

Jahn, F. S., Skovbye, M., Obenhausen, K., Jespersen, A. E., \& Miskowiak, K. W. (2021). Cognitive Training with Fully Immersive Virtual Reality in Patients with Neurological and Psychiatric Disorders: A Systematic Review of Randomized Controlled Trials. Psychiatry Research, 300, Article ID: 113928.

https://doi.org/10.1016/j.psychres.2021.113928

Joiner, T. E. (2005). Why People Die by Suicide. Harvard University Press.

Kessler, R. C., Berglund, P., Demler, O., Jin, R., Merikangas, K. R., \& Walters, E. E. (2005). Lifetime Prevalence and Age-of-Onset Distributions of DSM-IV Disorders in the National Comorbidity Survey Replication. Archives of General Psychiatry, 62, 593-602. https://doi.org/10.1001/archpsyc.62.6.593

Kessler, R. C., Mickelson, K. D., \& Williams, D. R. (1999). The Prevalence, Distribution, and Mental Health Correlates of Perceived Discrimination in the United States. Journal of Health and Social Behavior, 40, 208-230. https://doi.org/10.2307/2676349

Kessler, R. C., Sonnega, A., Bromet, E., Hughes, M., \& Nelson, C. B. (1995). Posttraumatic Stress Disorder in the National Comorbidity Survey. Archives of General Psychiatry, 52, 1048-1060. https://doi.org/10.1001/archpsyc.1995.03950240066012

Kira, I. (2004). Secondary Trauma in Treating Refugee Survivors of Torture and Their Families. Torture, 14, 38-44.

Kira, I. (2006). Collective Identity Terror in the Israeli-Palestinian Conflict and Potential Solutions. In J. Kuriansky (Ed.), Terror in the Holy Land, inside the Anguish of Israeli-Palestinian Conflict (pp. 125-130). Praeger.

Kira, I. (2010). Etiology and Treatments of Post-Cumulative Traumatic Stress Disorders in Different Cultures. Traumatology: An International Journal, 16, 128-141. https://doi.org/10.1177/1534765610365914

Kira, I. (2019). Toward an Integrative Theory of Self Identity and Identity Stressors and Traumas and Their Mental Health Dynamics. Psychology, 10, 385-410. https://doi.org/10.4236/psych.2019.104027

Kira, I. A. (2001). Taxonomy of Trauma and Trauma Assessment. Traumatology, 7, 73-86. https://doi.org/10.1177/153476560100700202

Kira, I. A. (2020). Refugee Status and Identity Development. In The Encyclopedia of Child and Adolescent Development. Wiley. https://doi.org/10.1002/9781119171492.wecad467

Kira, I. A. (2021). Taxonomy of Stressors and Traumas: An Update of the DevelopmentBased Trauma Framework (DBTF): A Life-Course Perspective on Stress and Trauma. Traumatology. https://doi.org/10.1037/trm0000305

Kira, I. A., \& Shuwiekh, H. (2021). Discrimination and Mental Health of Christians in Egypt: Coping Trajectories and Perceived Posttraumatic Growth. Mental Health, Religion \& Culture, 24, 1-22. https://doi.org/10.1080/13674676.2020.1832454

Kira, I. A., Alawneh, A. W. N., Aboumediane, S., Mohanesh, J., Ozkan, B., \& Alamia, H. (2011). Identity Salience and Its Dynamics in Palestinians Adolescents. Psychology, 2, 781. https://doi.org/10.4236/psych.2011.28120

Kira, I. A., Ashby, J. S., Omidy, A. Z., \& Lewandowski, L. (2015a). Current, Continuous, 
and Cumulative Trauma-Focused Cognitive Behavior Therapy: A New Model for Trauma Counseling. Journal of Mental Health Counseling, 37, 323-340.

https://doi.org/10.17744/mehc.37.4.04

Kira, I. A., Ayna, Y. E., Shuwiekh, H. A., \& Ashby, J. S. (2021c). The Association of WTELS as a Master Motivator with Higher Executive Functioning and Better Mental Health. Current Psychology, 1-12. https://doi.org/10.1007/s12144-021-02078-8

Kira, I. A., Fawzi, M., Shuwiekh, H., Lewandowski, L., Ashby, J. S., \& Al Ibraheem, B. (2019b). Do Adding Attachment, Oppression, Cumulative, and Proliferation Trauma Dynamics to PTSD Criterion "a” Improve Its Predictive Validity: Toward a Paradigm Shift? Current Psychology, 1-15. https://doi.org/10.1007/s12144-019-00206-Z

Kira, I. A., Lewandowski, L., Chiodo, L., \& Ibrahim, A. (2014a). Advances in Systemic Trauma Theory: Traumatogenic Dynamics and Consequences of Backlash as a Multi-Systemic Trauma on Iraqi Refugee Muslim Adolescents. Psychology, 5, 389-412. https://doi.org/10.4236/psych.2014.55050

Kira, I. A., Omidy, A. Z., \& Ashby, J. S. (2014b). Cumulative Trauma, Appraisal, and Coping in Palestinian and American Indian Adults: Two Cross-Cultural Studies. Traumatology: An International Journal, 20, 119-133. https://doi.org/10.1037/h0099397

Kira, I. A., Omidy, A. Z., Fawzi, M., Rice, K. G., Fawzi, M., Lewandowski, L., \& Bujold-Bugeaud, M. (2015b). Are the Negative Mental Health Effects of Gender Discrimination (GD) Salient across Cultures? Does Self-Esteem Mediate These Effects: GD as Continuous Traumatic Stress and the Pathways to Its Negative Dynamics? Psychology, 6, 93. https://doi.org/10.4236/psych.2015.61009

Kira, I. A., Özcan, N. A., Shuwiekh, H., Kucharska, J., Amthal, H. A. H., \& Kanaan, A. (2020b). The Cross-National Validity and Structural Invariance of the Existential Annihilation Anxiety Scale. Current Psychology, 1-12. https://doi.org/10.1007/s12144-019-00591-5

Kira, I. A., Shuweikh, H., Al-Huwailiah, A., El-Wakeel, S. A., Waheep, N. N., Ebada, E. E., \& Ibrahim, E. S. R. (2020d). The Direct and Indirect Impact of Trauma Types and Cumulative Stressors and Traumas on Executive Functions. Applied Neuropsychology: Adult, 1-17. https://doi.org/10.1080/23279095.2020.1848835

Kira, I. A., Shuwiekh, H. A., Alhuwailah, A., Ashby, J. S., Sous Fahmy Sous, M., Baali, S. B. A., Jamil, H. J. et al. (2020c). The Effects of COVID-19 and Collective Identity Trauma (Intersectional Discrimination) on Social Status and Well-Being. Traumatology, 27, 29-39. https://doi.org/10.1037/trm0000289

Kira, I. A., Shuwiekh, H., Al-Huwailah, A. H., Lewandowski, L., Alawneh, A.-W. N., Abou-Mediene, S., Al Ibraheem, B., \& Aljakoub, J. (2019d). The Central Role of Social Identity in Oppression, Discrimination, and Social-Structural Violence: Collective Identity Stressors and Traumas, Their Dynamics and Mental Health Impact. Peace and Conflict: Journal of Peace Psychology, 25, 262-268. https://doi.org/10.1037/pac0000363

Kira, I. A., Shuwiekh, H., Ashby, J. S., Rice, K., \& Alhuwailah, A. (2021f). Measuring COVID-19 Stressors and Their Impact: The Second-Order Factor Model and Its Four First-Order Factors: Infection Fears, Economic, Grief, and Lockdown Stressors. Journal of Loss and Trauma: International Perspectives on Stress \& Coping. https://doi.org/10.1080/15325024.2021.1920270

Kira, I. A., Shuwiekh, H., Rice, K., Al Ibraheem, B., \& Aljakoub, J. (2017). A Threatened Identity: The Mental Health Status of Syrian Refugees in Egypt and Its Etiology. Identity, 17, 176-190. https://doi.org/10.1080/15283488.2017.1340163

Kira, I. A., Templin, T., Lewandowski, L., \& Shuwiekh, H. (2018b). A Conceptual Model and Measurement of Identity-Based, Existential Annihilation Anxieties (EAA). Psy- 
chology, 9, 1306-1328. https://doi.org/10.4236/psych.2018.96080

Kira, I. A., Templin, T., Lewandowski, L., Ashby, J. S., Oladele, A., \& Odenat, L. (2012c). Cumulative Trauma Disorder Scale (CTD): Two Studies. Psychology, 3, 643-656. https://doi.org/10.4236/psych.2012.39099

Kira, I., Alpay, E. H., Ayna, W. E., Shuwiekh, H., Ashby, J. S., \& Turkeli, A. (2021a). The Effects of COVID-19 Continuous Traumatic Stressors on Mental Health and Cognitive Functioning: A Case Example from Turkey. Current Psychology. https://doi.org/10.1007/s12144-021-01743-2

Kira, I., Alpay, E. H., Turkeli, A., Shuwiekh, H., Ashby, J. S., \& Alhuwailah, A. (2021b). The Effects of COVID-19 Traumatic Stress on Executive Functions: The Case of Syrian Refugees in Turkey. Journal of Loss and Trauma: International Perspectives on Stress \& Coping, 26, 666-687. https://doi.org/10.1080/15325024.2020.1869444

Kira, I., Ashby, J. S., Lewandowski, L., Alawneh, A. N., Mohanesh, J., \& Odenat, L. (2013a). Advances in Continuous Traumatic Stress Theory: Traumatogenic Dynamics and Consequences of Intergroup Conflict: The Palestinian Adolescents' Case. Psychology, 4, 396-409. https://doi.org/10.4236/psych.2013.44057

Kira, I., Barger, B., Shuwiekh, H., Kucharska, J., \& Al-Huwailah, A. H. (2019c). Cumulative Stressors and Traumas and Suicide: A Non-Linear Cusp Dynamic Systems Model. Psychology, 10, 1999-2018. https://doi.org/10.4236/psych.2019.1015128

Kira, I., Barger, B., Shuwiekh, H., Kucharska, J., \& Al-Huwailah, A. (2020a). The Threshold Non-Linear Model for the Effects of Cumulative Stressors and Traumas: A Chained Cusp Catastrophe Analysis. Psychology, 11, 385-403.

https://doi.org/10.4236/psych.2020.113025

Kira, I., Fawzi, M., \& Fawzi, M. M. (2013b). The Dynamics of Cumulative Trauma and Trauma Types in Adult Patients with Psychiatric Disorders: Two Cross-Cultural Studies. Traumatology, 19, 179-195. https://doi.org/10.1177/1534765612459892

Kira, I., Lewandowski, L., Templin, T., Ramaswamy, V., Ozkan, B., \& Mohanesh, J. (2008a). Measuring Cumulative Trauma Dose, Types, and Profiles Using a Development-Based Taxonomy of Trauma. Traumatology, 14, 62-87. https://doi.org/10.1177/1534765608319324

Kira, I., Lewandowski, L., Yoon, J., Somers, C., \& Chiodo, L. (2012a). The Linear and Nonlinear Associations between Multiple Types of Trauma and IQ Discrepancy Indexes in African American and Iraqi Refugee Adolescents. Journal of Child \& Adolescent Trauma, 5, 47-62. https://doi.org/10.1080/19361521.2012.633239

Kira, I., Özcan, N. A., Shuwiekh, H., Kucharska, J., Al-Huwailah, A. H., \& Bujold-Bugeaud, M. (2021d). Mental Health Dynamics of Interfaith Spirituality in Believers and NonBelievers: The Two Circuit Pathways Model of Coping with Adversities: Interfaith Spirituality and Will-to Exist, Live and Survive. Psychology, 12, 992-1024. https://doi.org/10.4236/psych.2021.126060

Kira, I., Shuwiekh, H., Ashby, J.S., Elwakeel, S., Alhuwailah, A., Sous, M., Baali, S., Azdaou, C., Oliemat, E., \& Jamil, H. (2021e). The Impact of COVID-19 Traumatic Stressors on Mental Health: Is COVID-19 a New Trauma Type. International Journal of Mental health and Addiction. https://doi.org/10.1007/s11469-021-00577-0

Kira, I., Shuwiekh, H., Kucharska, J., \& Al-Huwailah, A. (2019c). The Integrated Structural and Measurement Models of Existential Annihilation Anxieties (EAA) and Their Potential Contribution to Clinical Science: Two Studies on Western and Non-Western Samples. Psychology, 10, 449-480. https://doi.org/10.4236/psych.2019.104031

Kira, I., Shuwiekh, H., Kucharska, J., Fawzi, M., Ashby, J. S., Omidy, A. Z., Abou-Mediene, S., \& Lewandowski, L. (2018b). Trauma Proliferation and Stress Generation (TPSG) 
Dynamics and Their Implications for Clinical Science. American Journal of Orthopsychiatry, 88, 582-596. https://doi.org/10.1037/ort0000304

Kira, I., Shuwiekh, H., Rice, K., Ashby, J. S., Alhuwailah, A., Sous, M., Baali, S., Azdaou, C., Oliemat, E., \& Jamil, H. (2021g). Coping with COVID-19 Continuous Complex Stressors: The "Will-to-Exist-Live, and Survive" and Perfectionistic Striving. Traumatology: An International Journal. https://doi.org/10.1037/trm0000352

Kira, I., Somers, C., Lewandowski, L., \& Chiodo, L. (2012b). Attachment Disruptions, IQ and PTSD in African American Adolescents (AAA): A Traumatology Perspective. Journal of Aggression, Maltreatment, and Trauma, 21, 665-690. https://doi.org/10.1080/10926771.2012.698377

Kira, I., Templin, T., Lewandowski, L., Ramaswamy, V., Bulent, O., Mohanesh, J., \& Abdulkhaleq, H. (2012d). Collective and Personal Annihilation Anxiety: Measuring Annihilation Anxiety AA. Psychology, 3, 90-99. https://doi.org/10.4236/psych.2012.31015

Kira, I., Templin, T., Lewandowski, L., Ramaswamy, V., Ozkan, B., \& Mohanesh, J. (2008b). Which Trauma Is the Worse of Them All? The Effects of Collective Identity Trauma/Racism and Discrimination. International Journal of Psychology, 43, 318.

Kroenke, K., Spitzer, R. L., \& Williams, J. B. (2001). The PHQ-9: Validity of a Brief Depression Severity Measure. Journal of General Internal Medicine, 16, 606-613.

Kucharska, J. (2018). Cumulative Trauma, Gender Discrimination and Mental Health in Women: The Mediating Role of Self-Esteem. Journal of Mental Health, 27, 416-423. https://doi.org/10.1080/09638237.2017.1417548

Lee, I. A., \& Preacher, K. J. (2013). Calculation for the Test of the Difference between Two Dependent Correlations with One Variable in Common [Computer Software].

Lewis, T. T., Aiello, A. E., Leurgans, S., Kelly, J., \& Barnes, L. L. (2010). Self-Reported Experiences of Everyday Discrimination Are Associated with Elevated C-Reactive Protein Levels in Older African-American Adults. Brain, Behavior, and Immunity, 24, 438-443. https://doi.org/10.1016/j.bbi.2009.11.011

Lindert, J., von Ehrenstein, O. S., Grashow, R., Gal, G., Braehler, E., \& Weisskopf, M. G. (2014). Sexual and Physical Abuse in Childhood Is Associated with Depression and Anxiety over the Life Course: Systematic Review and Meta-Analysis. International Journal of Public Health, 59, 359-372. https://doi.org/10.1007/s00038-013-0519-5

Luby, J., \& Kertz, S. (2019). Increasing Suicide Rates in Early Adolescent Girls in the United States and the Equalization of Sex Disparity in Suicide: The Need to Investigate the Role of Social Media. JAMA Network Open, 2, e193916.

https://doi.org/10.1001/jamanetworkopen.2019.3916

Malarbi, S., Abu-Rayya, H. M., Muscara, F., \& Stargatt, R. (2017). Neuropsychological Functioning of Childhood Trauma and Post-Traumatic Stress Disorder: A Meta-Analysis. Neuroscience \& Biobehavioral Reviews, 72, 68-86.

https://doi.org/10.1016/j.neubiorev.2016.11.004

McCann, I. L., \& Pearlman, L. A. (1990). Vicarious Trauma: A Framework for Understanding the Psychological Effects of Working with Victims. Journal of Traumatic Stress, 3, 131-149. https://doi.org/10.1007/BF00975140

McClendon, J., Chang, K., Boudreaux, M., Oltmanns, T. F., \& Bogdan, R. (2021). BlackWhite Racial Health Disparities in Inflammation and Physical Health: Cumulative Stress, Social Isolation, and Health Behaviors. Psychoneuroendocrinology, 131, Article ID: 105251. https://doi.org/10.1016/j.psyneuen.2021.105251

McKay, M. T., Cannon, M., Chambers, D., Conroy, R. M., Coughlan, H., Dodd, P., Clarke, M. C. et al. (2021). Childhood Trauma and Adult Mental Disorder: A Systematic Review and Meta-Analysis of Longitudinal Cohort Studies. Acta Psychiatrica 
Scandinavica, 143, 189-205. https://doi.org/10.1111/acps.13268

McLaughlin, K. A., Koenen, K. C., Hill, E. D., Petukhova, M., Sampson, N. A., Zaslavsky, A. M., \& Kessler, R. C. (2013). Trauma Exposure and Posttraumatic Stress Disorder in a National Sample of Adolescents. Journal of the American Academy of Child and Adolescent Psychiatry, 52, 815-830.e14. https://doi.org/10.1016/j.jaac.2013.05.011

Meade, A. W., \& Lautenschlager, G. J. (2004). A Comparison of Item Response Theory and Confirmatory Factor Analytic Methodologies for Establishing Measurement Equivalence/Invariance. Organizational Research Methods, 7, 361-388. https://doi.org/10.1177/1094428104268027

Milner, A., Page, A., \& LaMontagne, A. D. (2014). Cause and Effect in Studies on Unemployment, Mental Health, and Suicide: A Meta-Analytic and Conceptual Review. Psychological Medicine, 44, 909-917. https://doi.org/10.1017/S0033291713001621

Noelke, C., \& Avendano, M. (2015). Who Suffers during Recessions? Economic Downturns, Job Loss, and Cardiovascular Disease in Older Americans. American Journal of Epidemiology, 182, 873-882. https://doi.org/10.1093/aje/kwv094

Pallini, S., Chirumbolo, A., Morelli, M., Baiocco, R., Laghi, F., \& Eisenberg, N. (2018). The Relation of Attachment Security Status to Effortful Self-Regulation: A Meta-Analysis. Psychological Bulletin, 144, 501. https://doi.org/10.1037/bul0000134

Pallini, S., Morelli, M., Chirumbolo, A., Baiocco, R., Laghi, F., \& Eisenberg, N. (2019). Attachment and Attention Problems: A Meta-Analysis. Clinical Psychology Review, 74, Article ID: 101772. https://doi.org/10.1016/j.cpr.2019.101772

Pascoe, E. A., \& Richman, L. S. (2009). Perceived Discrimination and Health: A MetaAnalytic Review. Psychological Bulletin, 135, 531-554. https://doi.org/10.1037/a0016059

Pearlin, L. I., Aneshensel, C. S., \& LeBlanc, A. J. (1997). The Forms and Mechanisms of Stress Proliferation: The Case of AIDS Caregivers. Journal of Health and Social Behavior, 38, 223-236. https://doi.org/10.2307/2955368

Peh, O. H., Rapisarda, A., \& Lee, J. (2019). Childhood Adversities in People at Ultra-High Risk (UHR) for Psychosis: A Systematic Review and Meta-Analysis. Psychological Medicine, 49, 1089-1101. https://doi.org/10.1017/S003329171800394X

Peyrot, W. J., Van der Auwera, S., Milaneschi, Y., Dolan, C. V., Madden, P. A., Sullivan, P. F., Yang, J. et al. (2018). Does Childhood Trauma Moderate Polygenic Risk for Depression? A Meta-Analysis of 5765 Subjects from the Psychiatric Genomics Consortium. Biological Psychiatry, 84, 138-147. https://doi.org/10.1016/j.biopsych.2017.09.009

Plotkin-Amrami, G., \& Brunner, J. (2015). Making Up "National Trauma” in Israel: From Collective Identity to Collective Vulnerability. Social Studies of Science, 45, 525-545. https://doi.org/10.1177/0306312715589846

Pontifex, M. B., Parks, A. C., Paoli, A. G. D., Schroder, H. S., \& Moser, J. S. (2021). The Effect of Acute Exercise for Reducing Cognitive Alterations Associated with Individuals High in Anxiety. International Journal of Psychophysiology.

https://doi.org/10.1016/j.ijpsycho.2021.06.008

Roelfs, D. J., Shor, E., Davidson, K. W., \& Schwartz, J. E. (2011). Losing Life and Livelihood: A Systematic Review and Meta-Analysis of Unemployment and All-Cause Mortality. Social Science \& Medicine, 72, 840-854.

https://doi.org/10.1016/j.socscimed.2011.01.005

Rönnblad, T., Grönholm, E., Jonsson, J., Koranyi, I., Orellana, C., Kreshpaj, B., Bodin, T. et al. (2019). Precarious Employment and Mental Health: A Systematic Review and Meta-Analysis of Longitudinal Studies. Scandinavian Journal of Work, Environment \& Health, 45, 429-443. https://doi.org/10.5271/sjweh.3797 
Sawaya, H., Atoui, M., Hamadeh, A., Zeinoun, P., \& Nahas, Z. (2016). Adaptation and Initial Validation of the Patient Health Questionnaire-9 (PHQ-9) and the Generalized Anxiety Disorder-7 Questionnaire (GAD-7) in an Arabic Speaking Lebanese Psychiatric Outpatient Sample. Psychiatry Research, 239, 245-252.

Schmitt, M. T., Branscombe, N. R., Postmes, T., \& Garcia, A. (2014). The Consequences of Perceived Discrimination for Psychological Well-Being: A Meta-Analytic Review. Psychological Bulletin, 140, 921-948. https://doi.org/10.1037/a0035754

Seng, J. S., Lopez, W. D., Sperlich, M., Hamama, L., \& Meldrum, C. D. R. (2012). Marginalized Identities, Discrimination Burden, and Mental Health: Empirical Exploration of an Interpersonal-Level Approach to Modeling Intersectionality. Social Science \& Medicine, 75, 2437-2445. https://doi.org/10.1016/j.socscimed.2012.09.023

Shneidman, E. S. (1993). Commentary: Suicide as Psychache. Journal of Nervous and Mental Disease, 181, 147-149. https://doi.org/10.1097/00005053-199303000-00001

Snyder, H. R., Friedman, N. P., \& Hankin, B. L. (2020). Associations between Task Performance and Self-Report Measures of Cognitive Control: Shared versus Distinct Abilities. Assessment, 28, 1080-1096. https://doi.org/10.1177/1073191120965694

Solomon, E. P., \& Heide, K. M. (1999). Type III Trauma: Toward a More Effective Conceptualization of Psychological Trauma. International Journal of Offender Therapy and Comparative Criminology, 43, 202-210. https://doi.org/10.1177/0306624X99432007

Spencer, C., Mallory, A. B., Cafferky, B. M., Kimmes, J. G., Beck, A. R., \& Stith, S. M. (2019). Mental Health Factors and Intimate Partner Violence Perpetration and Victimization: A Meta-Analysis. Psychology of Violence, 9, 1-17.

https://doi.org/10.1037/vio0000156

Spinazzola, J., Van der Kolk, B., \& Ford, J. D. (2018). When Nowhere Is Safe: Interpersonal Trauma and Attachment Adversity as Antecedents of Posttraumatic Stress Disorder and Developmental Trauma Disorder. Journal of Traumatic Stress, 31, 631-642. https://doi.org/10.1002/jts.22320

Spinazzola, J., Van der Kolk, B., \& Ford, J. D. (2021). Developmental Trauma Disorder: A Legacy of Attachment Trauma in Victimized Children. Journal of Traumatic Stress. https://doi.org/10.1002/jts.22320

Spitzer, R. L., Kroenke, K., Williams, J. B., \& Löwe, B. (2006). A Brief Measure for Assessing Generalized Anxiety Disorder: The GAD-7. Archives of Internal Medicine, 166, 1092-1097.

Spruit, A., Goos, L., Weenink, N., Rodenburg, R., Niemeyer, H., Stams, G. J., \& Colonnesi, C. (2020). The Relation between Attachment and Depression in Children and Adolescents: A Multilevel Meta-Analysis. Clinical Child and Family Psychology Review, 23, 54-69. https://doi.org/10.1007/s10567-019-00299-9

Steele, H., \& Steele, M. (2017). Handbook of Attachment-Based Interventions. Guilford Publications.

Steiger, J. H. (1980). Tests for Comparing Elements of a Correlation Matrix. Psychological Bulletin, 87, 245-251. https://doi.org/10.1037/0033-2909.87.2.245

Stryker, S., \& Serpe, R. T. (1994). Identity Salience and Psychological Centrality: Equivalent, Overlapping, or Complementary Concepts? Social Psychology Quarterly, 57, 16-35. https://doi.org/10.2307/2786972

Sue, D. W., \& Spanierman, L. (2020). Microaggressions in Everyday Life. John Wiley \& Sons.

Terr, L. C. (1995). Childhood Traumas. In G. S. Everly, \& J. M. Lating (Eds.), Psychotraumatology: Key Papers and Core Concepts in Post-Traumatic Stress (pp. 301-320). 
Plenum Press. https://doi.org/10.1007/978-1-4899-1034-9 18

Tosone, C., Nuttman-Shwartz, O., \& Stephens, T. (2012). Shared Trauma: When the Profession Is Personal. Clinical Social Work Journal, 40, 231-239. https://doi.org/10.1007/s10615-012-0395-0

Trickey, D., Siddaway, A. P., Meiser-Stedman, R., Serpell, L., \& Field, A. P. (2012). A Meta-Analysis of Risk Factors for Post-Traumatic Stress Disorder in Children and Adolescents. Clinical Psychology Review, 32, 122-138. https://doi.org/10.1016/j.cpr.2011.12.001

Umaña-Taylor, A. J., \& Douglass, S. (2017). Developing an Ethnic-Racial Identity Intervention from a Developmental Perspective: Process, Content, and Implementation of the Identity Project. In Handbook on Positive Development of Minority Children and Youth (pp. 437-453). Springer. https://doi.org/10.1007/978-3-319-43645-6 26

Varese, F., Smeets, F., Drukker, M., Lieverse, R., Lataster, T., Viechtbauer, W., Bentall, R. P. et al. (2012). Childhood Adversities Increase the Risk of Psychosis: A Meta-Analysis of Patient-Control, Prospective-and Cross-Sectional Cohort Studies. Schizophrenia Bulletin, 38, 661-671. https://doi.org/10.1093/schbul/sbs050

Vargas, S. M., Huey, S. J. Jr., \& Miranda, J. (2020). A Critical Review of Current Evidence on Multiple Types of Discrimination and Mental Health. American Journal of Orthopsychiatry, 90, 374-390. https://doi.org/10.1037/ort0000441

Vasileva, M., \& Petermann, F. (2018). Attachment, Development, and Mental Health in Abused and Neglected Preschool Children in Foster Care: A Meta-Analysis. Trauma, Violence, \& Abuse, 19, 443-458. https://doi.org/10.1177/1524838016669503

Williams, D. R., \& Mohammed, S. A. (2009). Discrimination and Racial Disparities in Health: Evidence and Needed Research. Journal of Behavioral Medicine, 32, 20-47. https://doi.org/10.1007/s10865-008-9185-0

Woodhouse, S., Ayers, S., \& Field, A. P. (2015). The Relationship between Adult Attachment Style and Post-Traumatic Stress Symptoms: A Meta-Analysis. Journal of Anxiety Disorders, 35, 103-117. https://doi.org/10.1016/j.janxdis.2015.07.002

Zahodne, L. B., Kraal, A. Z., Sharifian, N., Zaheed, A. B., \& Sol, K. (2019). Inflammatory Mechanisms Underlying the Effects of Everyday Discrimination on Age-Related Memory Decline. Brain, Behavior, and Immunity, 75, 149-154. https://doi.org/10.1016/j.bbi.2018.10.002

Zahodne, L. B., Sharifian, N., Kraal, A. Z., Zaheed, A. B., Sol, K., Morris, E. P., Schupf, N., Manly, J. J., \& Brickman, A. M. (2021). Socioeconomic and Psychosocial Mechanisms Underlying Racial/Ethnic Disparities in Cognition among Older Adults. Neuropsychology, 35, 265-275. https://doi.org/10.1037/neu0000720

Zatti, C., Rosa, V., Barros, A., Valdivia, L., Calegaro, V. C., Freitas, L. H., Schuch, F. B. et al. (2017). Childhood Trauma and Suicide Attempt: A Meta-Analysis of Longitudinal Studies from the Last Decade. Psychiatry Research, 256, 353-358. https://doi.org/10.1016/j.psychres.2017.06.082

Zheng, L., Luo, Y., \& Chen, X. (2020). Different Effects of Attachment Anxiety and Attachment Avoidance on Depressive Symptoms: A Meta-Analysis. Journal of Social and Personal Relationships, 37, 3028-3050. https://doi.org/10.1177/0265407520946482 


\section{Appendix 1}

\section{Cumulative Trauma Scale (Short Form)}

\section{Directions:}

Many people have experienced different kinds of events and situations in their lives. The following questions will ask you about some specific events; please indicate how many times happened if happened and how much it affected you negatively or positively on the provided scale.

1) In my life, I witnessed or experienced natural disasters, e.g. earthquakes, hurricanes, tornados, or floods.
Never
once two times
three times many times.

- If this happened, how old you were when the first one happened?

- If this happened, how has this affected you?

\begin{tabular}{ccccccc}
\hline $\begin{array}{c}\text { Extremely } \\
\text { positive }\end{array}$ & Very positive & $\begin{array}{c}\text { Somewhat } \\
\text { positive }\end{array}$ & Neutral & $\begin{array}{c}\text { Somewhat } \\
\text { Negative }\end{array}$ & Very Negative & $\begin{array}{c}\text { Extremely } \\
\text { Negative }\end{array}$ \\
\hline $\mathbf{1}$ & $\mathbf{2}$ & $\mathbf{3}$ & $\mathbf{4}$ & $\mathbf{5}$ & $\mathbf{6}$ & $\mathbf{7}$ \\
\hline
\end{tabular}

2) I have experienced a life-threatening accident, e.g., motor vehicle accidents.
Never
once
two times
three times many times.

- If this happened, how old you were when the first one happened?

- If this happened, how has this affected you?

\begin{tabular}{ccccccc}
\hline $\begin{array}{c}\text { Extremely } \\
\text { positive }\end{array}$ & Very positive & $\begin{array}{c}\text { Somewhat } \\
\text { positive }\end{array}$ & Neutral & $\begin{array}{c}\text { Somewhat } \\
\text { Negative }\end{array}$ & Very Negative & $\begin{array}{c}\text { Extremely } \\
\text { Negative }\end{array}$ \\
\hline $\mathbf{1}$ & $\mathbf{2}$ & $\mathbf{3}$ & $\mathbf{4}$ & $\mathbf{5}$ & $\mathbf{6}$ & $\mathbf{7}$ \\
\hline
\end{tabular}

3) I have been involved in or exposed to war or combat.

Never once two times three times many times.

- If this happened, how old you were when the first one happened?

- If this happened, how has this affected you?

\begin{tabular}{ccccccc}
\hline $\begin{array}{c}\text { Extremely } \\
\text { positive }\end{array}$ & Very positive & $\begin{array}{c}\text { Somewhat } \\
\text { positive }\end{array}$ & Neutral & $\begin{array}{c}\text { Somewhat } \\
\text { Negative }\end{array}$ & Very Negative & $\begin{array}{c}\text { Extremely } \\
\text { Negative }\end{array}$ \\
\hline $\mathbf{1}$ & $\mathbf{2}$ & $\mathbf{3}$ & $\mathbf{4}$ & $\mathbf{5}$ & $\mathbf{6}$ & $\mathbf{7}$ \\
\hline
\end{tabular}

4) I have experienced the sudden death of one of my parents or a close friend or loved one.

Never once two times three times many times.

- If this happened, how old you were when the first one happened?

- If this happened, how much this affected you:

\begin{tabular}{ccccccc}
\hline $\begin{array}{c}\text { Extremely } \\
\text { positive }\end{array}$ & Very positive & $\begin{array}{c}\text { Somewhat } \\
\text { positive }\end{array}$ & Neutral & $\begin{array}{c}\text { Somewhat } \\
\text { Negative }\end{array}$ & Very Negative & $\begin{array}{c}\text { Extremely } \\
\text { Negative }\end{array}$ \\
\hline $\mathbf{1}$ & $\mathbf{2}$ & $\mathbf{3}$ & $\mathbf{4}$ & $\mathbf{5}$ & $\mathbf{6}$ & $\mathbf{7}$ \\
\hline
\end{tabular}

5) I have experienced a life-threatening or permanently disabling event for loved ones (e.g., parents, close friends). 
Never once two times three times many times.

- If this happened, how old you were when the first one happened?

- If this happened how, has this affected you?

\begin{tabular}{ccccccc}
\hline $\begin{array}{c}\text { Extremely } \\
\text { positive }\end{array}$ & Very positive & $\begin{array}{c}\text { Somewhat } \\
\text { positive }\end{array}$ & Neutral & $\begin{array}{c}\text { Somewhat } \\
\text { Negative }\end{array}$ & Very Negative & $\begin{array}{c}\text { Extremely } \\
\text { Negative }\end{array}$ \\
\hline $\mathbf{1}$ & $\mathbf{2}$ & $\mathbf{3}$ & $\mathbf{4}$ & $\mathbf{5}$ & $\mathbf{6}$ & $\mathbf{7}$ \\
\hline
\end{tabular}

6) I have experienced life-threatening illness or permanently disabling event (e.g., cancer, stroke, serious chronic illness, or major injury).

Never once two times three times many times.

- If this happened, how old you were when the first one happened?

- If this happened, how has this affected you?

\begin{tabular}{cccccccc}
\hline $\begin{array}{c}\text { Extremely } \\
\text { positive }\end{array}$ & Very positive & $\begin{array}{c}\text { Somewhat } \\
\text { positive }\end{array}$ & Neutral & $\begin{array}{c}\text { Somewhat } \\
\text { Negative }\end{array}$ & Very Negative & $\begin{array}{c}\text { Extremely } \\
\text { Negative }\end{array}$ \\
\hline $\mathbf{1}$ & $\mathbf{2}$ & $\mathbf{3}$ & $\mathbf{4}$ & $\mathbf{5}$ & $\mathbf{6}$ & $\mathbf{7}$ \\
\hline
\end{tabular}

7) I have experienced robbery involving a weapon (robbed or mugged).

\section{Never once two times three times many times.}

- If this happened, how old you were when the first one happened?

- If this happened, how much this affected you:

\begin{tabular}{ccccccc}
\hline $\begin{array}{c}\text { Extremely } \\
\text { positive }\end{array}$ & Very positive & $\begin{array}{c}\text { Somewhat } \\
\text { positive }\end{array}$ & Neutral & $\begin{array}{c}\text { Somewhat } \\
\text { Negative }\end{array}$ & Very Negative & $\begin{array}{c}\text { Extremely } \\
\text { Negative }\end{array}$ \\
\hline $\mathbf{1}$ & $\mathbf{2}$ & $\mathbf{3}$ & $\mathbf{4}$ & $\mathbf{5}$ & $\mathbf{6}$ & $\mathbf{7}$ \\
\hline
\end{tabular}

8) I witnessed a severe assault of an acquaintance or stranger (e.g., got shot, stabbed, or severely beaten up).

Never once two times three times many times

- If this happened, how old you were when the first one happened?

- If this happened, how has this affected you?

\begin{tabular}{ccccccc}
\hline $\begin{array}{c}\text { Extremely } \\
\text { positive }\end{array}$ & Very positive & $\begin{array}{c}\text { Somewhat } \\
\text { positive }\end{array}$ & Neutral & $\begin{array}{c}\text { Somewhat } \\
\text { Negative }\end{array}$ & Very Negative & $\begin{array}{c}\text { Extremely } \\
\text { Negative }\end{array}$ \\
\hline $\mathbf{1}$ & $\mathbf{2}$ & $\mathbf{3}$ & $\mathbf{4}$ & $\mathbf{5}$ & $\mathbf{6}$ & $\mathbf{7}$ \\
\hline
\end{tabular}

9) I have been threatened to be killed or to be seriously harmed.

Never once two times three times many times.

- If this happened, how old you were when the first one happened?

- If this happened, how has this affected you?

\begin{tabular}{ccccccc}
\hline $\begin{array}{c}\text { Extremely } \\
\text { positive }\end{array}$ & Very positive & $\begin{array}{c}\text { Somewhat } \\
\text { positive }\end{array}$ & Neutral & $\begin{array}{c}\text { Somewhat } \\
\text { Negative }\end{array}$ & Very Negative & $\begin{array}{c}\text { Extremely } \\
\text { Negative }\end{array}$ \\
\hline $\mathbf{1}$ & $\mathbf{2}$ & $\mathbf{3}$ & $\mathbf{4}$ & $\mathbf{5}$ & $\mathbf{6}$ & $\mathbf{7}$ \\
\hline
\end{tabular}


10) I have been physically abused, pushed hard enough to cause injury, or beaten up by a caretaker, e.g., parent...

Never once two times three times many times.

- If this happened, how old you were when the first one happened?

- If this happened, how has this affected you?

\begin{tabular}{cccccccc}
\hline $\begin{array}{c}\text { Extremely } \\
\text { positive }\end{array}$ & Very positive & $\begin{array}{c}\text { Somewhat } \\
\text { positive }\end{array}$ & Neutral & $\begin{array}{c}\text { Somewhat } \\
\text { Negative }\end{array}$ & Very Negative & $\begin{array}{c}\text { Extremely } \\
\text { Negative }\end{array}$ \\
\hline $\mathbf{1}$ & $\mathbf{2}$ & $\mathbf{3}$ & $\mathbf{4}$ & $\mathbf{5}$ & $\mathbf{6}$ & $\mathbf{7}$ \\
\hline
\end{tabular}

11) I witnessed heard one of my parents or caregivers hitting, hurting, and threatening to kill my other parent or caregiver.

Never once two times three times many times.

- If this happened, how old you were when the first one happened?

- If this happened, how has this affected you?

\begin{tabular}{cccccccc}
\hline $\begin{array}{c}\text { Extremely } \\
\text { positive }\end{array}$ & Very positive & $\begin{array}{c}\text { Somewhat } \\
\text { positive }\end{array}$ & Neutral & $\begin{array}{c}\text { Somewhat } \\
\text { Negative }\end{array}$ & Very Negative & $\begin{array}{c}\text { Extremely } \\
\text { Negative }\end{array}$ \\
\hline $\mathbf{1}$ & $\mathbf{2}$ & $\mathbf{3}$ & $\mathbf{4}$ & $\mathbf{5}$ & $\mathbf{6}$ & $\mathbf{7}$ \\
\hline
\end{tabular}

12) I was led to sexual contact with someone older than me.

Never once two times three times many times.

- If this happened, how old you were when the first one happened?

- If this happened, how has this affected you?

\begin{tabular}{ccccccc}
\hline $\begin{array}{c}\text { Extremely } \\
\text { positive }\end{array}$ & Very positive & $\begin{array}{c}\text { Somewhat } \\
\text { positive }\end{array}$ & Neutral & $\begin{array}{c}\text { Somewhat } \\
\text { Negative }\end{array}$ & Very Negative & $\begin{array}{c}\text { Extremely } \\
\text { Negative }\end{array}$ \\
\hline $\mathbf{1}$ & $\mathbf{2}$ & $\mathbf{3}$ & $\mathbf{4}$ & $\mathbf{5}$ & $\mathbf{6}$ & $\mathbf{7}$ \\
\hline
\end{tabular}

13) I was sexually abused or raped or involved in unwanted sex with one or more persons.

Never once two times three times many times.

- If this happened, how old you were when the first one happened?

- If this happened, how has this affected you?

\begin{tabular}{ccccccc}
\hline $\begin{array}{c}\text { Extremely } \\
\text { positive }\end{array}$ & Very positive & $\begin{array}{c}\text { Somewhat } \\
\text { positive }\end{array}$ & Neutral & $\begin{array}{c}\text { Somewhat } \\
\text { Negative }\end{array}$ & Very Negative & $\begin{array}{c}\text { Extremely } \\
\text { Negative }\end{array}$ \\
\hline $\mathbf{1}$ & $\mathbf{2}$ & $\mathbf{3}$ & $\mathbf{4}$ & $\mathbf{5}$ & $\mathbf{6}$ & $\mathbf{7}$ \\
\hline
\end{tabular}

14) I have been jailed and/or tortured.

Never once two times three times many times.

- If this happened, how old you were when the first one happened?

- If this happened, how has this affected you?

\begin{tabular}{ccccccc}
\hline $\begin{array}{c}\text { Extremely } \\
\text { positive }\end{array}$ & Very positive & $\begin{array}{c}\text { Somewhat } \\
\text { positive }\end{array}$ & Neutral & $\begin{array}{c}\text { Somewhat } \\
\text { Negative }\end{array}$ & Very Negative & $\begin{array}{c}\text { Extremely } \\
\text { Negative }\end{array}$ \\
\hline $\mathbf{1}$ & $\mathbf{2}$ & $\mathbf{3}$ & $\mathbf{4}$ & $\mathbf{5}$ & $\mathbf{6}$ \\
\hline DOI: $10.4236 /$ psych.2021.1210099 & & 1605 & & Psychology
\end{tabular}


15) My mother had abandoned or left/or separated from me when I was very young.

Never once two times three times many times.

- If this happened, how old you were when the first one happened?

- If this happened, how has this affected you?

\begin{tabular}{cccccccc}
\hline $\begin{array}{c}\text { Extremely } \\
\text { positive }\end{array}$ & Very positive & $\begin{array}{c}\text { Somewhat } \\
\text { positive }\end{array}$ & Neutral & $\begin{array}{c}\text { Somewhat } \\
\text { Negative }\end{array}$ & Very Negative & $\begin{array}{c}\text { Extremely } \\
\text { Negative }\end{array}$ \\
\hline $\mathbf{1}$ & $\mathbf{2}$ & $\mathbf{3}$ & $\mathbf{4}$ & $\mathbf{5}$ & $\mathbf{6}$ & $\mathbf{7}$ \\
\hline
\end{tabular}

16) My father had abandoned or left me or separated from me when I was young.

Never once two times three times many times.

- If this happened, how old you were when the first one happened?

- If this happened, how has this affected you?

\begin{tabular}{ccccccc}
\hline $\begin{array}{c}\text { Extremely } \\
\text { positive }\end{array}$ & Very positive & $\begin{array}{c}\text { Somewhat } \\
\text { positive }\end{array}$ & Neutral & $\begin{array}{c}\text { Somewhat } \\
\text { Negative }\end{array}$ & Very Negative & $\begin{array}{c}\text { Extremely } \\
\text { Negative }\end{array}$ \\
\hline $\mathbf{1}$ & $\mathbf{2}$ & $\mathbf{3}$ & $\mathbf{4}$ & $\mathbf{5}$ & $\mathbf{6}$ & $\mathbf{7}$ \\
\hline
\end{tabular}

17) I was put down, threatened, or discriminated against by some others' negative attitudes, stereotypes, or actions because of my ethnicity, race, culture, religion, or national origin.

Never once two times three times many times.

- If this happened, how old you were when the first one happened?

- If this happened, how has this affected you?

\begin{tabular}{ccccccc}
\hline $\begin{array}{c}\text { Extremely } \\
\text { positive }\end{array}$ & Very positive & $\begin{array}{c}\text { Somewhat } \\
\text { positive }\end{array}$ & Neutral & $\begin{array}{c}\text { Somewhat } \\
\text { Negative }\end{array}$ & Very Negative & $\begin{array}{c}\text { Extremely } \\
\text { Negative }\end{array}$ \\
\hline $\mathbf{1}$ & $\mathbf{2}$ & $\mathbf{3}$ & $\mathbf{4}$ & $\mathbf{5}$ & $\mathbf{6}$ & $\mathbf{7}$ \\
\hline
\end{tabular}

18) My parents went through divorce and or separation.

Never once two times three times many times.

- If this happened, how old you were when the first one happened?

- If this happened, how has this affected you?

\begin{tabular}{ccccccc}
\hline $\begin{array}{c}\text { Extremely } \\
\text { positive }\end{array}$ & Very positive & $\begin{array}{c}\text { Somewhat } \\
\text { positive }\end{array}$ & Neutral & $\begin{array}{c}\text { Somewhat } \\
\text { Negative }\end{array}$ & Very Negative & $\begin{array}{c}\text { Extremely } \\
\text { Negative }\end{array}$ \\
\hline $\mathbf{1}$ & $\mathbf{2}$ & $\mathbf{3}$ & $\mathbf{4}$ & $\mathbf{5}$ & $\mathbf{6}$ & $\mathbf{7}$ \\
\hline
\end{tabular}

19) My race has a history of being oppressed, discriminated against, or threatened by genocide.

Never (1) little like it (2) partially like it (3) moderately like it (4) very much like it (5)

- If this happened, how old you were when the first was aware of it?

- If this happened, how has this affected you?

\begin{tabular}{ccccccc}
\hline $\begin{array}{c}\text { Extremely } \\
\text { positive }\end{array}$ & Very positive & $\begin{array}{c}\text { Somewhat } \\
\text { positive }\end{array}$ & Neutral & $\begin{array}{c}\text { Somewhat } \\
\text { Negative }\end{array}$ & Very Negative & $\begin{array}{c}\text { Extremely } \\
\text { Negative }\end{array}$ \\
\hline $\mathbf{1}$ & $\mathbf{2}$ & $\mathbf{3}$ & $\mathbf{4}$ & $\mathbf{5}$ & $\mathbf{6}$ & $\mathbf{7}$ \\
\hline DOI: $10.4236 /$ psych.2021.1210099 & & & & & & \\
\hline
\end{tabular}


20) I have experienced a nervous breakdown or felt that I was about to have one (e.g., about to lose control) due to seemingly small but recurrent or unremitting hassles or chronic stressors.

\section{Never once two times three times many times.}

- If this happened, how old you were when the first one happened?

- If this happened, how has this affected you?

\begin{tabular}{ccccccc}
\hline $\begin{array}{c}\text { Extremely } \\
\text { positive }\end{array}$ & Very positive & $\begin{array}{c}\text { Somewhat } \\
\text { positive }\end{array}$ & Neutral & $\begin{array}{c}\text { Somewhat } \\
\text { Negative }\end{array}$ & Very Negative & $\begin{array}{c}\text { Extremely } \\
\text { Negative }\end{array}$ \\
\hline $\mathbf{1}$ & $\mathbf{2}$ & $\mathbf{3}$ & $\mathbf{4}$ & $\mathbf{5}$ & $\mathbf{6}$ & $\mathbf{7}$ \\
\hline
\end{tabular}

21) At least one of my parents or siblings was involved in war, combat, or being tortured.

Never once two times three times many times.

- If this happened, how old you were when the first one happened?

- If this happened, how has this affected you?

\begin{tabular}{ccccccc}
\hline $\begin{array}{c}\text { Extremely } \\
\text { positive }\end{array}$ & Very positive & $\begin{array}{c}\text { Somewhat } \\
\text { positive }\end{array}$ & Neutral & $\begin{array}{c}\text { Somewhat } \\
\text { Negative }\end{array}$ & Very Negative & $\begin{array}{c}\text { Extremely } \\
\text { Negative }\end{array}$ \\
\hline 1 & $\mathbf{2}$ & $\mathbf{3}$ & $\mathbf{4}$ & $\mathbf{5}$ & $\mathbf{6}$ & $\mathbf{7}$ \\
\hline
\end{tabular}

22) I experienced frequent failures in school.

Never once two times three times many times.

- If this happened, how old you were when the first one happened?

- If this happened, how has this affected you?

\begin{tabular}{ccccccc}
\hline $\begin{array}{c}\text { Extremely } \\
\text { positive }\end{array}$ & Very positive & $\begin{array}{c}\text { Somewhat } \\
\text { positive }\end{array}$ & Neutral & $\begin{array}{c}\text { Somewhat } \\
\text { Negative }\end{array}$ & Very Negative & $\begin{array}{c}\text { Extremely } \\
\text { Negative }\end{array}$ \\
\hline $\mathbf{1}$ & $\mathbf{2}$ & $\mathbf{3}$ & $\mathbf{4}$ & $\mathbf{5}$ & $\mathbf{6}$ & $\mathbf{7}$ \\
\hline
\end{tabular}

23) I was uprooted and forced to move from my intimate environment in town, village, or country.

Never once two times three times many times.

- If this happened, how old you were when the first one happened?

- If this happened, how has this affected you?

\begin{tabular}{ccccccc}
\hline $\begin{array}{c}\text { Extremely } \\
\text { positive }\end{array}$ & Very positive & $\begin{array}{c}\text { Somewhat } \\
\text { positive }\end{array}$ & Neutral & $\begin{array}{c}\text { Somewhat } \\
\text { Negative }\end{array}$ & Very Negative & $\begin{array}{c}\text { Extremely } \\
\text { Negative }\end{array}$ \\
\hline 1 & 2 & 3 & 4 & 5 & $\mathbf{6}$ & 7 \\
\hline
\end{tabular}

24) I have been physically attacked, beaten up by another stronger person or group of persons, and caused an injury.

Never once two times three times many times.

- If this happened, how old you were when the first one happened?

- If this happened, how has this affected you? 


\begin{tabular}{ccccccc}
\hline $\begin{array}{c}\text { Extremely } \\
\text { positive }\end{array}$ & Very positive & $\begin{array}{c}\text { Somewhat } \\
\text { positive }\end{array}$ & Neutral & $\begin{array}{c}\text { Somewhat } \\
\text { Negative }\end{array}$ & Very Negative & $\begin{array}{c}\text { Extremely } \\
\text { Negative }\end{array}$ \\
\hline $\mathbf{1}$ & $\mathbf{2}$ & $\mathbf{3}$ & $\mathbf{4}$ & $\mathbf{5}$ & $\mathbf{6}$ & $\mathbf{7}$ \\
\hline
\end{tabular}

25) I was led to sexual contact with one of my caregivers/parents.

\section{Never once two times three times many times.}

- If this happened, how old you were when the first one happened?

- If this happened, how has this affected you?

\begin{tabular}{ccccccc}
\hline $\begin{array}{c}\text { Extremely } \\
\text { positive }\end{array}$ & Very positive & $\begin{array}{c}\text { Somewhat } \\
\text { positive }\end{array}$ & Neutral & $\begin{array}{c}\text { Somewhat } \\
\text { Negative }\end{array}$ & Very Negative & $\begin{array}{c}\text { Extremely } \\
\text { Negative }\end{array}$ \\
\hline $\mathbf{1}$ & $\mathbf{2}$ & $\mathbf{3}$ & $\mathbf{4}$ & $\mathbf{5}$ & $\mathbf{6}$ & $\mathbf{7}$ \\
\hline
\end{tabular}

26) I was put down, denied my rights, or discriminated against in the society (not by family members), by some others' negative attitudes, stereotypes, or behaviors, or by institutions because of my gender (being a girl/woman or a boy/man).

Never once two times three times many times.

- If this happened, how old you were when the first time you become aware of it?

- If this happened, how has this affected you?

\begin{tabular}{ccccccc}
\hline $\begin{array}{c}\text { Extremely } \\
\text { positive }\end{array}$ & Very positive & $\begin{array}{c}\text { Somewhat } \\
\text { positive }\end{array}$ & Neutral & $\begin{array}{c}\text { Somewhat } \\
\text { Negative }\end{array}$ & Very Negative & $\begin{array}{c}\text { Extremely } \\
\text { Negative }\end{array}$ \\
\hline $\mathbf{1}$ & $\mathbf{2}$ & $\mathbf{3}$ & $\mathbf{4}$ & $\mathbf{5}$ & $\mathbf{6}$ & $\mathbf{7}$ \\
\hline
\end{tabular}

27) I experienced serious rejection or failure in my relationships.

Never once two times three times many times.

- If this happened, how old you were when the first one happened?

- If this happened, how has this affected you?

\begin{tabular}{ccccccc}
\hline $\begin{array}{c}\text { Extremely } \\
\text { positive }\end{array}$ & Very positive & $\begin{array}{c}\text { Somewhat } \\
\text { positive }\end{array}$ & Neutral & $\begin{array}{c}\text { Somewhat } \\
\text { Negative }\end{array}$ & Very Negative & $\begin{array}{c}\text { Extremely } \\
\text { Negative }\end{array}$ \\
\hline $\mathbf{1}$ & $\mathbf{2}$ & $\mathbf{3}$ & $\mathbf{4}$ & $\mathbf{5}$ & $\mathbf{6}$ & $\mathbf{7}$ \\
\hline
\end{tabular}

28) I experienced the loss of a child or spouse.

Never once two times three times many times.

- If this happened, how old you were when the first one happened?

- If this happened, how has this affected you?

\begin{tabular}{cccccccc}
\hline $\begin{array}{c}\text { Extremely } \\
\text { positive }\end{array}$ & Very positive & $\begin{array}{c}\text { Somewhat } \\
\text { positive }\end{array}$ & Neutral & $\begin{array}{c}\text { Somewhat } \\
\text { Negative }\end{array}$ & Very Negative & $\begin{array}{c}\text { Extremely } \\
\text { Negative }\end{array}$ \\
\hline $\mathbf{1}$ & $\mathbf{2}$ & $\mathbf{3}$ & $\mathbf{4}$ & $\mathbf{5}$ & $\mathbf{6}$ & $\mathbf{7}$
\end{tabular}

29) I experienced sudden unexpected employment termination, been laid off, or failed in business or big market loss. 
Never once two times three times many times.

- If this happened, how old you were when the first one happened?

- If this happened, how has this affected you?

\begin{tabular}{ccccccc}
\hline $\begin{array}{c}\text { Extremely } \\
\text { positive }\end{array}$ & Very positive & $\begin{array}{c}\text { Somewhat } \\
\text { positive }\end{array}$ & Neutral & $\begin{array}{c}\text { Somewhat } \\
\text { Negative }\end{array}$ & Very Negative & $\begin{array}{c}\text { Extremely } \\
\text { Negative }\end{array}$ \\
\hline $\mathbf{1}$ & $\mathbf{2}$ & $\mathbf{3}$ & $\mathbf{4}$ & $\mathbf{5}$ & $\mathbf{6}$ & $\mathbf{7}$ \\
\hline
\end{tabular}

30) I remarried.

Never once two times three times many times.

- If this happened, how old you were when the first one happened?

- If this happened, how has this affected you?

\begin{tabular}{ccccccc}
\hline $\begin{array}{c}\text { Extremely } \\
\text { positive }\end{array}$ & Very positive & $\begin{array}{c}\text { Somewhat } \\
\text { positive }\end{array}$ & Neutral & $\begin{array}{c}\text { Somewhat } \\
\text { Negative }\end{array}$ & Very Negative & $\begin{array}{c}\text { Extremely } \\
\text { Negative }\end{array}$ \\
\hline $\mathbf{1}$ & $\mathbf{2}$ & $\mathbf{3}$ & $\mathbf{4}$ & $\mathbf{5}$ & $\mathbf{6}$ & $\mathbf{7}$ \\
\hline
\end{tabular}

31) I experienced being part of a low-income family with many hardships.
0 Never poor
(1) somewhat poor
(2) poor
(3) very poor
(4) extremely poor

- If this happened, how old you were when the first time you were aware of it?

- If this happened, how has this affected you?

\begin{tabular}{cccccccc}
\hline $\begin{array}{c}\text { Extremely } \\
\text { positive }\end{array}$ & Very positive & $\begin{array}{c}\text { Somewhat } \\
\text { positive }\end{array}$ & Neutral & $\begin{array}{c}\text { Somewhat } \\
\text { Negative }\end{array}$ & Very Negative & $\begin{array}{c}\text { Extremely } \\
\text { Negative }\end{array}$ \\
\hline $\mathbf{1}$ & $\mathbf{2}$ & $\mathbf{3}$ & $\mathbf{4}$ & $\mathbf{5}$ & $\mathbf{6}$ & $\mathbf{7}$ \\
\hline
\end{tabular}

32) I was put down, threatened, or discriminated against by some other family members (e.g., parents, siblings) negative attitudes, stereotypes, or actions because of my gender: being a boy or girl.

Never once two times three times many times.

- If this happened, how old you were when the first you experienced it?

- If this happened, how has this affected you?

\begin{tabular}{cccccccc}
\hline $\begin{array}{c}\text { Extremely } \\
\text { positive }\end{array}$ & Very positive & $\begin{array}{c}\text { Somewhat } \\
\text { positive }\end{array}$ & Neutral & $\begin{array}{c}\text { Somewhat } \\
\text { Negative }\end{array}$ & Very Negative & $\begin{array}{c}\text { Extremely } \\
\text { Negative }\end{array}$ \\
\hline $\mathbf{1}$ & $\mathbf{2}$ & $\mathbf{3}$ & $\mathbf{4}$ & $\mathbf{5}$ & $\mathbf{6}$ & $\mathbf{7}$ \\
\hline
\end{tabular}

33) I have had to harm another person.

\section{Never once two times three times many times.}

- If this happened, how old you were when the first one happened?

- If this happened, how has this affected you?

\begin{tabular}{|c|c|c|c|c|c|c|}
\hline $\begin{array}{l}\text { Extremely } \\
\text { positive }\end{array}$ & Very positive & $\begin{array}{l}\text { Somewhat } \\
\text { positive }\end{array}$ & Neutral & $\begin{array}{l}\text { Somewhat } \\
\text { Negative }\end{array}$ & Very Negative & $\begin{array}{c}\text { Extremely } \\
\text { Negative }\end{array}$ \\
\hline DI: $10.4236 / p$ & 2021.1210099 & & 1609 & & & Psycho \\
\hline
\end{tabular}


34) I lived in a community full of violence and criminal activities.

Never once two times three times many times.

- If this happened, how old you were when the first one happened?

- If this happened, how has this affected you?

\begin{tabular}{|c|c|c|c|c|c|c|}
\hline $\begin{array}{l}\text { Extremely } \\
\text { positive }\end{array}$ & Very positive & $\begin{array}{l}\text { Somewhat } \\
\text { positive }\end{array}$ & Neutral & $\begin{array}{c}\text { Somewhat } \\
\text { Negative }\end{array}$ & Very Negative & $\begin{array}{c}\text { Extremely } \\
\text { Negative }\end{array}$ \\
\hline
\end{tabular}

35) I have been discriminated against because of my sexual preference.

Never once two times three times many times.

- If this happened, how old you were when the first one happened?

- If this happened, how has this affected you?

\begin{tabular}{|c|c|c|c|c|c|c|}
\hline $\begin{array}{l}\text { Extremely } \\
\text { positive }\end{array}$ & Very positive & $\begin{array}{c}\text { Somewhat } \\
\text { positive }\end{array}$ & Neutral & $\begin{array}{c}\text { Somewhat } \\
\text { Negative }\end{array}$ & Very Negative & $\begin{array}{c}\text { Extremely } \\
\text { Negative }\end{array}$ \\
\hline
\end{tabular}

36) I have been told that my birth was complicated.

(0)Never (2) difficult (3)complicated (4)very complicated (5)extremely (life-threatening)

- If this happened, how old you were when you were told of it?

- If this happened, how had this affected you?

\begin{tabular}{|c|c|c|c|c|c|c|}
\hline $\begin{array}{l}\text { Extremely } \\
\text { positive }\end{array}$ & Very positive & $\begin{array}{l}\text { Somewhat } \\
\text { positive }\end{array}$ & Neutral & $\begin{array}{c}\text { Somewhat } \\
\text { Negative }\end{array}$ & Very Negative & $\begin{array}{c}\text { Extremely } \\
\text { Negative }\end{array}$ \\
\hline
\end{tabular}

\section{Instructions for Users with different age groups:}

- Children (age 8 - 11): delete items 14, 19, 28, 29 and 30,

- Adolescents (12 - 18 years old) delete items, 28, 29, and 30, interview the child or parents as appropriate,

- For younger children (age 1 - 7), you can interview one of the parents or the caregiver as appropriate.

- You can use all the parameters of the questions (occurrence, frequency, age, and appraisal) or only one or more according to the assessment and research goals.

- You can use the items and subscales or only selected subscales according to the assessment or research goals.

- The interview format is the preferred format for children and illiterate adults and adolescents. Paper and pencil format is the preferred format for literate adults and adolescents. (Interview format are plausible for all age groups based on the research design)

\section{Scoring}

Suggested Measures:

For each of the following suggested measures you can generate:

1) Occurrence scale (first parameter). (happened vs. not happened).

2) Frequency Scale (second parameter). (if happened how many times).

3) Negative Appraisal (recode appraisal: $5=1,6=2,7=3$ ).

4) Positive (or non-negative) Appraisal (recode appraisal: $4=1,3=2,2=3,1=4$ ). 
5) Age when the trauma first happened parameter.

\section{Subscales:}

Gender Discrimination $=26+32$

Personal identity trauma $=(12+13+25)+24+27+10$

A. Sexual abuse $=12+13+25$

B. Physical abuse $=10+24$

Collective identity Trauma (social structural violence) $=17+19(+31)+14+35+(26+32)$

A. Discrimination $=17+19+35(26+32)$

B. Poverty $=(31)$

Torture $=14$

Uprootedness $=23$

Attachment Trauma $=15+16$

Achievement (Role Identity) Trauma $=22+29$

Survival Traumas $=1+2+3+6+7+9$

Secondary Traumas $=4+5+8+11+18+21+28$

Cumulative Stressors and Traumas $=1-36$

Cumulative non-traumatic Stressors $=20,23,30$

Perpetration Trauma $=33$

Community Violence $=34$

Birthing Trauma $=36$

Measures for trauma types

Type 1 traumas

$1,2,4,7,8,28$ (6 items)

Type II Traumas

$3,10,12,13,14,15,16,22,24,25$, (10 items)

Type III Traumas

$6,10,17,19,26,31,32,34,35,36$ (10 items)

Type IV Traumas $=1-36$

- Note: We excluded the non-traumatic chronic stressors and some of the secondary traumas from the trauma types to make the difference in intensity clear. For item 24 . If the physical abuse is one event, it is time 1 , if it was recurrent and the frequency of happening was more than one, then we consider it to be type II.

- Accordingly item $24^{*}$, If it was one event, then it is type 1 , if the frequency is more than one time, then it is type II.

\section{References}

Kira, I. (2001). Taxonomy of Trauma and Trauma Assessment. Traumatology, 2, 1-14. https://doi.org/10.1177/153476560100700202

Kira, I. A. (2021). Taxonomy of Stressors and Traumas: An Update of the Development-Based Trauma Framework (DBTF): A Life-Course Perspective on Stress and Trauma. Traumatology. https://doi.org/10.1037/trm0000305

Kira, I., Lewandowski, L., Templin, T., Ramaswamy, V., Ozkan, B., \& Mohanesh, J. (2008). Measuring Cumulative Trauma Dose, Types and Profiles Using a Development-Based Taxonomy of Trauma. Traumatology: International Journal, $14,62-87$. https://doi.org/10.1177/1534765608319324 


\section{Description of the Measure}

Cumulative stressors and traumas scale (CTS-S-36 items; Kira et al., 2008): CST-S is developed to measure stressors and traumas identified in the development-based stressors and traumas framework (DBTF) (e.g., Kira, 2001; Kira, 2019; Kira, 2021; Kira, Shuwiekh, et al., 2018; Kira, Shuwiekh, et al., 2019). The scale is intended to be a comprehensive measure of cumulative stressors and trauma exposure. Cumulative non-traumatic stressors included the severe life changes associated with widowed/ divorce and re-marrying, the significant life changes in forced relocations (e.g., uprootedness and immigration), and the experience of seemingly minor but recurrent or unremitting hassles or chronic stressors. The scale includes, in addition to cumulative non-traumatic stressors (3 items), seven major trauma types (acute stressors): collective identity trauma (3 items), personal identity trauma (6 items), survival trauma (6 items), attachment trauma (2 items), secondary trauma (7 items), achievement traumas (2 items) and gender discrimination ( 2 items). Recently, the scale developers added three items that measure: perpetration trauma, community violence, and birthing trauma (complicated birth) as optional items for interested researchers. Collective identity trauma includes trauma-related exposure to war and torture and discrimination based on race, ethnicity, or national origin. Personal identity trauma includes trauma related to sexual abuse, rape, incest, and being robbed. Attachment trauma includes abandonment by parents. Survival trauma includes car accidents, life-threatening illnesses, and natural disasters. The achievement or role identity trauma is intended to measure traumatic stressors related to achieving life goals like success in school or business. Secondary trauma includes witnessing a traumatic event occurring to another individual or group and affecting social interdependence. Gender discrimination includes gender discrimination by parents (family) and gender discrimination by society and institutions. Gender discrimination items are worded to apply to both genders. In response to each item on the measure. The scale groups various traumas considering their severity to three types of traumas: types 1, II, and III traumas.

Additionally, the scale contains three items that assess chronic hassles and significant life non-acute stressors and subscale for intersected discrimination). The CST-S assesses cumulative stressors and traumas concerning their frequency, their mere occurrence, types, age of happening, and their appraisals (negative and positive). In this short study, we used only frequency and occurrence questions. In answering each question on the scale, participants were asked to describe their involvement with an event on a 5-point Likert-type scale ( $0=$ never; $4=$ many times). The CST-S consists of two overall measures for cumulative stressors and traumas' dose: occurrence and frequency. Researchers can compute subscales for each of the stressor/trauma types or use individual items as indicators of the experience of such an event. The CST-S has shown adequate internal consistency $(\alpha=.85)$, and test-retest stability (.95 in 4 weeks), and convergent, and divergent and predictive validity in different studies (e.g., Kira, Barger et al., 2019; Kira, Barger et al., 2020; Kira, Fawzi et al., 2013; Kira et al., 2019; Kira et al., 2018). The current alpha of cumulative stressors and traumatic occurrence is .97. In a further development, three optional items included community violence, birthing trauma (complicated birth), and perpetration trauma. Participants are instructed to indicate their experience with a traumatic event on a 5-point Likert-type scale ( $0=$ never; $4=$ many times). If a participant denotes that she/he has experienced the traumatic event, then he/she is asked to describe her/his appraisal of its effect on a 7-point Likert-type scale ( 1 = extremely positive; 7 = extremely negative). CTS-S includes two general subscales for cumulative trauma dose: occurrence and frequency of experience, and two appraisal subscales: negative and positive appraisal of events. These four sub-scales may also be generated for each of the trauma types.

The CTS-S has shown adequate internal consistency ( $\alpha=.85$; Kira et al., 2008, Kira, Fawzi et al., 2013). Evidence of the instrument's predictive validity includes cumulative trauma significantly predicting post-traumatic stress disorder $(\mathrm{r}=.54, p<.001)$, cumulative trauma-related disorders $(\mathrm{r}=.24, p<.001)$, and poor health $(\mathrm{r}=.37, p<.001$; Kira et al., 2008). CTS-S has also shown divergent validity: It was significantly negatively correlated with sociocultural adjustment $(\mathrm{r}=-.25, p<.001)$ and futuristic orientation $(\mathrm{r}=-.37, p<.001)$. CTS-S has been used with a variety of 
clinical and community samples of adults and adolescents from numerous sociocultural groups and has been shown to possess adequate reliability (with an alpha ranging between .80 and .92), good construct validity (e.g., Kira et al., 2008, Kira, Lewandowski, Somers, Yoon, \& Chiodo, 2012; Kira, Smith, Lewandowski, \& Templin, 2010), and validity across different cultural and clinical groups, including American Indians, Mayans, Palestinians, Egyptians, refugees, and torture survivors from 32 countries (e.g., Kira, Ashby, Odenat, \& Lewandowski, 2013; Kira, Barger et al., 2019, 2020; Kira, Fawzi et al., 2013; Kira, Omidy, \& Ashby, 2014; Kira et al., 2010). The measure has been used in several studies as a comprehensive measure of stress and trauma (e.g., Bedoya et al., 2020; Eltan, 2019; Gillespie \& Gates, 2013; Head, Singh, \& Bugg, 2012; Lee, \& Lee, 2018; Millender, 2013; Omidy, 2012; Rizeq, Flora, \& McCann, 2018; Robles et al., 2009), and has been found to have good reliability and predictive validity. It has been translated into different languages, including Arabic, Spanish, Polish, Korean, Turkish, and Nigerian, and proved to have good psychometric properties in these languages. Test-retest using an independent sample with four-week intervals yielded excellent stability coefficients (.995 for cumulative trauma frequency and .997 for cumulative trauma appraisal). The alpha for the main scale of occurrence was .88 in Egyptian data and .91 in Polish data.

\section{References}

Bedoya, E. Y., Ruíz, S., Córdoba, A., Rendón, G. D., Ruíz, G. I., \& Gómez, G. D.(2020) Traumatic Events and Psychopathological Symptoms in University Students. Revista de Psicopatología y Psicología Clínica, 1, 69-79. https://doi.org/10.5944/rppc.25786

Eltan, S. (2019). Psychometric Properties of the Cumulative Trauma Scale: Evaluation of the Reliability and Validity in a Turkish Sample. Thesis, Graduate School of Social Science, Middle East Technical University, Turkey.

Gillespie, G. L., \& Gates, D. M. (2013). Using Proactive Coping to Manage the Stress of Trauma Patient Care. Journal of Trauma Nursing, 20, 44-50. https://doi.org/10.1097/JTN.0b013e318286608e

Head, D., Singh, T., \& Bugg, J. M. (2012). The Moderating Role of Exercise on Stress-Related Effects on the Hippocampus and Memory in Later Adulthood. Neuropsychology, 26, 133. https://doi.org/10.1037/a0027108

Kira, I. (2001). Taxonomy of Trauma and Trauma Assessment. Traumatology, 2, 1-14. https://doi.org/10.1177/153476560100700202

Kira, I. (2010). Etiology and Treatments of Post-Cumulative Traumatic Stress Disorders in Different Cultures. Traumatology: An International Journal, 16, 128-141. https://doi.org/10.1177/1534765610365914

Kira, I. A., Omidy, A. Z., \& Ashby, J. S. (2014). Cumulative Trauma, Appraisal, and Coping in Palestinian and American Indian Adults: Two Cross-Cultural Studies. Traumatology: An International Journal, 20, 119-133. https://doi.org/10.1037/h0099397

Kira, I. A., Shuwiekh, H., Kucharska, J., Fawzi, M., Ashby, J. S., Omidy, A. Z., Abou-Mediene, S., \& Lewandowski, L. (2018). Trauma Proliferation and Stress Generation (TPSG) Dynamics and Their Implications for Clinical Science. American Journal of Orthopsychiatry, 88, 582-596. https://doi.org/10.1037/ort0000304

Kira, I., Ashby J. S., Lewandowski, L., Alawneh, A. N., Mohanesh, J., \& Odenat, L. (2013). Advances in Continuous Traumatic Stress Theory: Traumatogenic Dynamics and Consequences of Intergroup Conflict: The Palestinian Adolescents Case. Psychology, 4, 396-409. https://doi.org/10.4236/psych.2013.44057

Kira, I., Barger, B., Shuwiekh, H., Kucharska, J., \& Al-Huwailah, A. H. (2019). Cumulative Stressors and Traumas and Suicide: A Non-Linear Cusp Dynamic Systems Model. Psychology, 10, 1999-2018. https://doi.org/10.4236/psych.2019.1015128

Kira, I., Barger, B., Shuwiekh, H., Kucharska, J., \& Al-Huwailah, A. (2020). The Threshold Non-Linear Model for the Effects of Cumulative Stressors and Traumas: A Chained Cusp Catastrophe Analysis. Psychology, 11, 385-403. https://doi.org/10.4236/psych.2020.113025

Kira, I., Fawzi, M., \& Fawzi, M. (2013). The Dynamics of Cumulative Trauma and Trauma Types in Adults Patients with Psychiatric Disorders: Two Cross-Cultural Studies. Traumatology: An International Journal, 19, 179-195. https://doi.org/10.1177/1534765612459892 
Kira, I., Lewandowski, L., Chiodo, L., \& Ibrahim, A. (2014). Advances in Systemic Trauma Theory: Traumatogenic Dynamics and Consequences of Backlash as a Multi-Systemic Trauma on Iraqi Refugee Muslim Adolescents. Psychology, 5, 389-412. https://doi.org/10.4236/psych.2014.55050

Kira, I., Lewandowski, L., Somers, C., Yoon, J., \& Chiodo, L. (2012). PTSD, Trauma Types, Cumulative Trauma, and IQ: The Case of African American and Iraqi Refugee Adolescents. Psychological Trauma: Theory, Research, Practice, and Policy, 4, $128-139$. https://doi.org/10.1037/a0022121

Kira, I., Lewandowski, L., Templin, T., Ramaswamy, V., Ozkan, B., \& Mohanesh, J. (2008). Measuring Trauma Dose, Types, and Profiles Using a Development-Based Taxonomy of Trauma. Traumatology: International Journal, 14, 62-87. https://doi.org/10.1177/1534765608319324

Kira, I., Lewandowski, L., Templin, T., Ramaswamy, V., Ozkan, B., \& Mohanesh, J. (2008). Measuring Cumulative Trauma Dose, Types and Profiles Using a Development-Based Taxonomy of Trauma. Traumatology: International Journal, $14,62-87$. https://doi.org/10.1177/1534765608319324

Kira, I., Smith, I., Lewandowski, L., \& Templin, T. (2010).The Effects of Perceived Gender Discrimination on Refugee Torture Survivors: A Cross-Cultural Traumatology Perspective. Journal of the American Psychiatric Nurses Association, 16, $299-306$. https://doi.org/10.1177/1078390310384401

Lee, Y. K. C. J. H., \& Lee, H. P. L. H. S. (2018). Cognitive, Emotional, and Physiological Reactions to Mental Imagery as Predictors of Posttraumatic Stress Disorder. Korean Journal of Clinical Psychology, 37, 339-359.

https://doi.org/10.15842/kjcp.2018.37.3.007

Millender, E. I. (2013). Cumulative Trauma among Adult Mayas Living in Southeast Florida (196 p.). Ph.D. Dissertation, Florida Atlantic University.

Omidy, A. Z. (2012). Influence of Cumulative Trauma, Binge Eating, and Coping Styles on the General Health of American Indians. Doctoral Dissertation, University of Oklahoma.

Rizeq, J., Flora, D. B., \& McCann, D. (2018). Construct Validation of the Trauma Symptom Checklist-40 Total and Subscale Scores. Assessment, 27, 1016-1028.

Robles, M. E., Badosa, J. M., Roig, A., Pina, B., \& Feixas Viaplana, G. (2009). La evaluación del estrés y del trauma:Presentación de la versión española de la escala de trauma acumulativo (CTS). Revista de Psicoterapia, 20, 89-104.

https://doi.org/10.33898/rdp.v20i80.841 